\title{
Asymptotic expansions of certain $q$-series and a formula of Ramanujan for specific values of the Riemann zeta function
}

\author{
by \\ MASANORI KATSURADA (Yokohama) \\ Dedicated to Professor Iekata Shiokawa \\ on the occasion of his 60th birthday
}

1. Introduction. Throughout the present paper, $q$ is a complex parameter with $|q|<1$, and the substitution $q=e^{-t}$ will be made if necessary, transforming the half-plane Re $t>0$ to the unit disk $|q|<1$. It is the principal aim of the present paper to study intrinsic linkage between asymptotic expansions of certain $q$-series (see (1.6)-(1.8) below) and a formula of Ramanujan for specific values of the Riemann zeta function at odd integers (see (1.9)). This linkage is in fact hidden in Ramanujan's original work; however, the introduction of the $q$-series (1.2) or (1.3) and its treatment based on a Mellin transform technique (see (6.3)) suggest connecting these two aspects together. It is worth while noting that this technique is advantageous, from a heuristic point of view, in studying certain asymptotic aspects and transformation properties of zeta and theta functions (see [Ka1-Ka7]).

Let $z$ and $s$ be complex variables, and let $\alpha$ and $\lambda$ be real parameters with $\alpha>0$. For our later purposes it is convenient to introduce the generalized Lerch zeta function $\Phi(s, \alpha, z)$ defined by

$$
\Phi(s, \alpha, z)=\sum_{n=0}^{\infty}(\alpha+n)^{-s} z^{n}
$$

for all $s$ if $|z|<1$, for $\operatorname{Re} s>0$ if $|z|=1$ and $z \neq 1$, and for $\operatorname{Re} s>1$ if $z=1$,

2000 Mathematics Subject Classification: Primary 11M35; Secondary 11P82.

Key words and phrases: Riemann zeta function, Hurwitz zeta function, Lerch zeta function, $q$-series, theta series, Rogers-Ramanujan identities, Mellin transform, asymptotic expansion.

Supported in part by Grant-in-Aid for Scientific Research (No. 11640041), the Ministry of Education, Science, Sports and Culture of Japan. 
respectively; it continues to a meromorphic function over the whole $s$-plane and is one-valued in the complex $z$-plane cut along the real axis from 1 to $+\infty$ (cf. [Er1, 1.11, (1) and (5)]). We use the notation $e(\lambda)=e^{2 \pi i \lambda}$ hereafter. Then $\Phi(s, \alpha, z)$ reduces to the ordinary Lerch zeta function $\phi(s, \alpha, \lambda)$ when $z=e(\lambda)$, so that $\Phi(s, \alpha, 1)=\zeta(s, \alpha)$ is the Hurwitz zeta function, $e(\lambda) \Phi(s, 1, e(\lambda))=\zeta_{\lambda}(s)$ the exponential zeta function, and $\Phi(s, 1,1)=\zeta(s)$ the Riemann zeta function. We remark that the order of the variables in $\Phi$ and $\phi$ above differs from the usual notation, in order to retain notational consistency with other terminology.

Let $\beta$ and $\mu$ be real parameters with $\beta>0$. The main object of the present paper is the $q$-series of the form

$$
S_{s}(\alpha, \beta ; \lambda, \mu ; q)=e(\alpha \lambda+\beta \mu) \sum_{l=0}^{\infty} e(\lambda l) q^{(\alpha+l) \beta} \Phi\left(s, \beta, e(\mu) q^{\alpha+l}\right),
$$

which is rewritten, by changing the order of summation, in a Lambert series form

$$
S_{s}(\alpha, \beta ; \lambda, \mu ; q)=e(\alpha \lambda+\beta \mu) \sum_{m=0}^{\infty}(\beta+m)^{-s} \frac{e(\mu m) q^{\alpha(\beta+m)}}{1-e(\lambda) q^{\beta+m}},
$$

where the factor $e(\alpha \lambda+\beta \mu)$ is added to clarify the transformation properties of the $q$-series (see Theorems 3 and 4 in Section 4 ). We shall prove complete asymptotic expansions of $S_{s}(\alpha, \beta ; \lambda, \mu ; q)$ as $t \rightarrow 0$ in the sectorial region $|\arg t|<\pi / 2$ (see Theorem 0 below). As usual, let

$$
(z ; q)_{\infty}=\prod_{m=0}^{\infty}\left(1-z q^{m}\right), \quad(z ; q)_{n}=(z ; q)_{\infty} /\left(z q^{n} ; q\right)_{\infty}
$$

for any integer $n$ denote $q$-shifted factorials. Our main formula (2.3) in particular implies a complete asymptotic expansion of $\log \left(q^{\alpha} ; q\right)_{\infty}$ as $q \rightarrow$ $1-0$, and it further allows us to treat the $q$-series

$$
\begin{gathered}
F(q)=\sum_{n=0}^{\infty} \frac{q^{n^{2}}}{(q ; q)_{n}^{2}}, \\
G(q)=\sum_{n=0}^{\infty} \frac{q^{n^{2}}}{(q ; q)_{n}} \text { and } H(q)=\sum_{n=0}^{\infty} \frac{q^{n(n+1)}}{(q ; q)_{n}} .
\end{gathered}
$$

These are typical examples of theta series (in the transformed Eulerian form) whose asymptotic behaviour near the singularities at $q^{k}=1(k=1,2, \ldots)$ was first considered by Ramanujan in his last letter to Hardy (see [Wa]). Ramanujan showed

$$
F(q)=\left(\frac{t}{2 \pi}\right)^{1 / 2} \exp \left(\frac{\pi^{2}}{6 t}-\frac{t}{24}\right)+o(1)
$$




$$
\begin{aligned}
& G(q)=\left(\frac{2}{5-\sqrt{5}}\right)^{1 / 2} \exp \left(\frac{\pi^{2}}{15 t}-\frac{t}{60}\right)+o(1), \\
& H(q)=\left(\frac{2}{5+\sqrt{5}}\right)^{1 / 2} \exp \left(\frac{\pi^{2}}{15 t}+\frac{11 t}{60}\right)+o(1),
\end{aligned}
$$

as $t \rightarrow+0$, and similar asymptotic formulae for certain other $q$-series. In connection with this result, (complete) Stirling's formula for the $q$-gamma function was first established by Moak [Mo], while Ueno and Nishizawa [UN] developed their theory of a $q$-analogue of the Hurwitz zeta function and applied it to rederive the same formula, together with asymptotic expansions of $G(q)$ and $H(q)$, similar to (1.7) and (1.8). The study of asymptotic aspects for more general $q$-series of the type $\sum_{n=0}^{\infty} a^{n} q^{b n^{2}+c n} /(q ; q)_{n}$ was initiated by Ramanujan [Ra1, p. 366], [Ra2, p. 359], and was further continued by Berndt [Be3], [Be4, Chap. 27]. This direction has recently been systematically explored by McIntosh [Mc1-Mc3] and Gordon and McIntosh [GM1-GM2], together with transformation properties of the $q$-series. It is to be remarked that the basic tool applied by these authors is Euler-Maclaurin summation. The Mellin transform technique, on the other hand, was applied by Meinardus [Me1-Me2] to derive certain asymptotic formulae for a fairly general class of partition-type functions. We refer the reader to [An, Chap. 6, Notes] for related work.

Let $B_{k}(k=0,1,2, \ldots)$ denote the Bernoulli numbers (cf. [Er1, 1.13, (1)]). Our main theorem also yields Ramanujan's famous formula for specific values of the Riemann zeta function at odd integers (cf. [Be1, Theorem 2.4], [Be2, Chap. 14, Entry 21(i)]), which asserts that, for any integer $n \neq 0$,

$$
\begin{aligned}
\xi^{-n}\left\{\frac{1}{2} \zeta(2 n+1)+\sum_{l=1}^{\infty} \frac{l^{-2 n-1}}{e^{2 l \xi}-1}\right\} \\
+2^{2 n} \sum_{k=0}^{n+1} \frac{B_{2 n+2-2 k} B_{2 k}}{(2 n+2-2 k) !(2 k) !} \xi^{n+1-k}(-\eta)^{k} \\
=(-\eta)^{-n}\left\{\frac{1}{2} \zeta(2 n+1)+\sum_{l=1}^{\infty} \frac{l^{-2 n-1}}{e^{2 l \eta}-1}\right\},
\end{aligned}
$$

where $\xi$ and $\eta$ are positive numbers satisfying $\xi \eta=\pi^{2}$ and the finite sum on the left-hand side is to be regarded as null if $n<-1$ (see Theorem 2 in Section 4). It will later turn out that the excluded case $n=0$ of this formula emerges (in a sense) as asymptotic expansions of $F(q), G(q)$ and $H(q)$ (see Corollary 1.4 in Section 3).

It is partly possible to formulate, within the framework of our method, the problem of investigating the asymptotic behaviour of the $q$-series near the singularities at $q^{k}=1(k=1,2, \ldots)$. Let $h$ be an integer relatively prime 
to $k$. We shall prove a complete asymptotic expansion of $\log \left(e(\mu) q^{\alpha} ; q\right)_{\infty}$ as $q \rightarrow e(h / k)$ in the unit disk, and take a closer look especially at the case of $\mu=0$ and $\alpha=1$ (see Theorem 5 in Section 5).

The present paper is organized as follows. The results are stated in Sections $2-5$, while the remaining sections are devoted to their proofs. Our main result (Theorem 0) is presented in the next section. In Section 3 an asymptotic expansion of $\log \left(e(\mu) q^{\alpha} ; q\right)_{\infty}$ as $q \rightarrow 1$ is first stated (Theorem 1), and its various applications are given (Corollaries 1.1-1.6) in connection with the Rogers-Ramanujan identities, Ramanujan's symmetric theta function $f(a, b)$ (see (3.15) below), the $q$-gamma function, the $q$-beta function and the $q$-hypergeometric functions. In Section 4 the connection between our main formula (2.3) and Ramanujan's formula for $\zeta(2 n+1)$ is established (Theorem 2), while deriving several variants of the latter (Theorems 3 and 4). Asymptotic expansions of $\log \left(e(\mu) q^{\alpha} ; q\right)_{\infty}$ as $q \rightarrow e(h / k)$ are stated (Theorem 5) in Section 5. The main theorem is proved in Section 6, and the remaining Sections 7, 8 and 9 are devoted to showing Theorem 1, Theorems $2-4$, Theorem 5 respectively, each together with its corresponding corollaries.

The author would like to thank the referee for valuable comments and refinements of the first version of the present paper.

2. The main theorem. Let $x$ and $y$ be complex variables. Apostol [Ap1] introduced the sequence of rational functions $\mathcal{B}_{k}(x, y)(k \geq 0)$ defined by the Taylor series expansion

$$
\frac{z e^{x z}}{y e^{z}-1}=\sum_{k=0}^{\infty} \frac{\mathcal{B}_{k}(x, y)}{k !} z^{k}
$$

near $z=0$. The function $\mathcal{B}_{k}(x, y)$, which coincides with the usual Bernoulli polynomial $B_{k}(x)$ if $y=1$, is a polynomial in $x$ of degree at most $k$ with coefficients in $\mathbb{Q}(y)$; further properties of $\mathcal{B}_{k}(x, y)$ will be given in Remark 2 of Theorem 0, and Lemmas 1, 2, 7 and 9 below. Next let $\Gamma(s)$ be the gamma function, and $\Psi(a, c ; z)$ the confluent hypergeometric function defined by

$$
\Psi(a, c ; z)=\frac{1}{\Gamma(a)} \int_{0}^{\infty e^{i \varphi}} e^{-z w} w^{a-1}(1+w)^{c-a-1} d w
$$

for $\operatorname{Re} a>0,-\pi<\varphi<\pi$ and $-\pi / 2<\arg z+\varphi<\pi / 2$, where the path of integration is taken as the half-line from the origin to $\infty e^{i \varphi}$ (cf. [Er1, 6.5, (3)]); the domain of $z$ is extended to the whole sector $|\arg z|<3 \pi / 2$ by rotating suitably the path of integration in (2.2).

Our main theorem can be stated as 
Theorem 0. Let $\alpha, \beta, \lambda$ and $\mu$ be real parameters with $\alpha>0$ and $\beta>0, q=e^{-t}$, and let $S_{s}(\alpha, \beta ; \lambda, \mu ; q)$ be defined by (1.2) or (1.3). Then for any integer $K \geq 0$ and any complex $t$ in the sector $|\arg t|<\pi / 2$,

$$
\begin{aligned}
S_{s}(\alpha, \beta ; \lambda, \mu ; q)= & e(\alpha \lambda+\beta \mu) \mathcal{B}_{0}(\beta, e(\mu)) \Gamma(1-s) \phi(1-s, \alpha, \lambda) t^{s-1} \\
& +e(\alpha \lambda+\beta \mu) \\
& \times \sum_{k=-1}^{K-1} \frac{(-1)^{k+1} \mathcal{B}_{k+1}(\alpha, e(\lambda))}{(k+1) !} \phi(s-k, \beta, \mu) t^{k} \\
& +R_{s, K}(\alpha, \beta ; \lambda, \mu ; q)
\end{aligned}
$$

in the region $\operatorname{Re} s<K+1$ except at $s=k(k=0,1, \ldots, K)$, where $\mathcal{B}_{k}(x, y)$ is defined by (2.1) and the empty sum is to be regarded as null. Here $R_{s, K}(\alpha, \beta ; \lambda, \mu ; q)$ is the remainder term satisfying the estimate

$$
R_{s, K}(\alpha, \beta ; \lambda, \mu ; q)=O\left(|t|^{K}\right)
$$

as $t \rightarrow 0$ through $|\arg t| \leq \pi / 2-\delta$ with any small $\delta>0$, in the region $\operatorname{Re} s<K+1$, where the implied $O$-constant depends at most on $s, K, \alpha$, $\beta, \lambda, \mu$ and $\delta$. In particular when $K \geq 1,0<\alpha, \beta \leq 1,0 \leq \lambda, \mu \leq 1$ the explicit expression

$$
\begin{aligned}
& \times\left\{e^{\pi i s / 2} \sum_{l, m=0}^{\infty} e(-\alpha l-\beta m)(\lambda+l)^{-s} f_{s, K}\left(4 \pi^{2} e^{-\pi i}(\lambda+l)(\mu+m) / t\right)\right. \\
& +e^{-\pi i s / 2} \sum_{l, m=0}^{\infty} e(\alpha(1+l)+\beta(1+m))(1-\lambda+l)^{-s} \\
& \times f_{s, K}\left(4 \pi^{2} e^{\pi i}(1-\lambda+l)(1-\mu+m) / t\right) \\
& +e^{\pi i s / 2} \sum_{l, m=0}^{\infty}{ }^{\prime} e(-\alpha l+\beta(1+m))(\lambda+l)^{-s} f_{s, K}\left(4 \pi^{2}(\lambda+l)(1-\mu+m) / t\right) \\
& +e^{-\pi i s / 2} \sum_{l, m=0}^{\infty} e(\alpha(1+l)-\beta m)(1-\lambda+l)^{-s} \\
& \left.\times f_{s, K}\left(4 \pi^{2}(1-\lambda+l)(\mu+m) / t\right)\right\}
\end{aligned}
$$

holds for $|\arg t|<\pi / 2$, in the region $\operatorname{Re} s<K$, where

$$
f_{s, K}(z)=\Psi(K+1-s, K+1-s ; z)
$$

with the confluent hypergeometric function defined by (2.2), and the primed summation symbols indicate that the terms including $\lambda+l=0$ or $1-\lambda+l$ $=0$, and $\mu+m=0$ or $1-\mu+m=0$ (if they occur) are to be omitted. 
Remark 1. Asymptotic expansions similar to (2.3) follow also for the exceptional points $s=k(k=0,1,2, \ldots)$ as limiting cases of Theorem 0 , whose important applications are included in these exceptional cases (see Theorems $1-5$ below).

REMARK 2. From the definition it is immediate that

$$
\begin{aligned}
& \mathcal{B}_{0}(x, y)= \begin{cases}1 & \text { if } y=1, \\
0 & \text { otherwise }\end{cases} \\
& \mathcal{B}_{1}(x, y)= \begin{cases}x-1 / 2 & \text { if } y=1, \\
1 /(y-1) & \text { otherwise. }\end{cases}
\end{aligned}
$$

REMARK 3. It is known that $\Psi(a, a ; z)$ can be evaluated in terms of the incomplete gamma function $\Gamma(1-a, z)$ (cf. [Er1, 6.9.2, (21)]); however, the use of the confluent hypergeometric notation is better fit for our purpose.

3. Applications to $q$-factorials and allied functions. First, from the relation $z \Phi(1,1, z)=-\log (1-z)$ for $|z|<1$ and (1.2), it is seen that

$$
S_{1}(\alpha, 1 ; 0, \mu ; q)=-\log \left(e(\mu) q^{\alpha} ; q\right)_{\infty},
$$

and hence Theorem 0 yields

Theorem 1. Let $\alpha>0$ and $0<\mu<1$. Then the following asymptotic expansions hold for any integer $K \geq 1$ and any complex $t$ with $|\arg t|<\pi / 2$ :

$$
\begin{aligned}
\log \left(q^{\alpha} ; q\right)_{\infty}= & -\frac{\pi^{2}}{6 t}-B_{1}(\alpha) \log t-\log \frac{\Gamma(\alpha)}{\sqrt{2 \pi}}+\frac{1}{4} B_{2}(\alpha) t \\
& -\sum_{k=2}^{K-1} \frac{(-1)^{k} B_{k} B_{k+1}(\alpha)}{k(k+1) !} t^{k}-R_{1, K}(\alpha, 1 ; 0,0 ; q) ; \\
\log \left(e(\mu) q^{\alpha} ; q\right)_{\infty}= & -\zeta_{\mu}(2) t^{-1}-B_{1}(\alpha)\left\{\log (2 \sin \pi \mu)+\pi i B_{1}(\mu)\right\} \\
& +\frac{1}{4} B_{2}(\alpha)(1-i \cot \pi \mu) t \\
& -\sum_{k=2}^{K-1} \frac{(-1)^{k} \mathcal{B}_{k}(0, e(\mu)) B_{k+1}(\alpha)}{k(k+1) !} t^{k} \\
& -R_{1, K}(\alpha, 1 ; 0, \mu ; q),
\end{aligned}
$$

where the remainder terms $R_{1, K}(\alpha, 1 ; 0,0 ; q)$ and $R_{1, K}(\alpha, 1 ; 0, \mu ; q)$ satisfy the same estimate as in (2.4) when $t \rightarrow 0$ through the sector $|\arg t| \leq \pi / 2-\delta$ with any small $\delta>0$. In particular, if $K \geq 2$ and $0<\alpha \leq 1$, the explicit expressions as in (2.5) follow for the remainder terms.

REMARK 1. In fact the terms with $k=2 h+1(h=1,2, \ldots)$ in $(3.2)$ do not appear in the asymptotic series by (3.6) below. 
Remark 2. A complete asymptotic expansion of $\left(q^{\alpha} ; q\right)_{\infty}$ as $q \rightarrow 1-0$ was first established by Moak [Mo] and later rederived by Ueno and Nishizawa [UN] in a form slightly different from (3.2). McIntosh [Mc1], [Mc3] proved (3.2) for $t>0$ with the error term $O\left(t^{K}\right)$ in a more general form.

The case $\mu=1 / 2$ of (3.3) reduces to

Corollary 1.1. For any $\alpha>0$ and any integer $K \geq 1$,

$$
\begin{aligned}
\log \left(-q^{\alpha} ; q\right)_{\infty}= & \frac{\pi^{2}}{12 t}-B_{1}(\alpha) \log 2+\frac{1}{4} B_{2}(\alpha) t \\
& -\sum_{k=2}^{K-1} \frac{(-1)^{k}\left(2^{k}-1\right) B_{k} B_{k+1}(\alpha)}{k(k+1) !} t^{k} \\
& -R_{1, K}(\alpha, 1 ; 0,1 / 2 ; q)
\end{aligned}
$$

for $|\arg t|<\pi / 2$, where the remainder term $R_{1, K}(\alpha, 1 ; 0,1 / 2 ; q)$ satisfies the same estimate as in (2.4). In particular, if $0<\alpha \leq 1$ and $K \geq 2$, the explicit expression as in (2.5) follows for the remainder term.

To describe the subsequent results, the change of the base

$$
q=e^{-t} \mapsto e^{-4 \pi^{2} / t}=\widehat{q}
$$

is frequently applied. Noting that

$$
\begin{gathered}
B_{2 h+1}=0, \quad h=1,2, \ldots, \\
B_{k}(1-\alpha)=(-1)^{k} B_{k}(\alpha), \quad k=0,1,2, \ldots
\end{gathered}
$$

(cf. [Er1, 1.13, (12) and (17)]), we find that every term (with $k \geq 2$ ) of the series in (3.2) and (3.4) vanishes when $\alpha=1$, and hence Theorem 0 further reduces to

COROLlary 1.2. The following formulae hold:

$$
\log (q ; q)_{\infty}=-\frac{\pi^{2}}{6 t}-\frac{1}{2} \log \frac{t}{2 \pi}+\frac{t}{24}-\sum_{l=1}^{\infty} l^{-1} \frac{\widehat{q}^{l}}{1-\widehat{q}^{l}},
$$

or in exponential form

$$
\begin{aligned}
(q ; q)_{\infty} & =\sqrt{\frac{2 \pi}{t}} \exp \left(-\frac{\pi^{2}}{6 t}+\frac{t}{24}\right)(\widehat{q} ; \widehat{q})_{\infty} \\
\log (-q ; q)_{\infty} & =\frac{\pi^{2}}{12 t}-\frac{1}{2} \log 2+\frac{t}{24}-\sum_{l=1}^{\infty} l^{-1} \frac{\widehat{q}^{l / 2}}{1-\widehat{q}^{l}}
\end{aligned}
$$

or in exponential form

$$
(-q ; q)_{\infty}=\frac{1}{\sqrt{2}} \exp \left(\frac{\pi^{2}}{12 t}+\frac{t}{24}\right)\left(\widehat{q}^{1 / 2} ; \widehat{q}\right)_{\infty}
$$


Remark 1. Formulae (3.8) and (3.9) are classic; they can be found for e.g., in [Ap2, Chap. 3].

Remark 2. Formulae (3.8) and (3.9) both give complete (convergent) asymptotic expansions, since for instance the $l$ th term of the last infinite series in (3.8) is of order $\widehat{q}^{l} / l+O\left(\widehat{q}^{2 l}\right)$ as $l \rightarrow \infty$.

It can be observed that the explicit expression (2.5) for the remainder term, in certain specific cases (as in the preceding corollary), further reduces to complete (convergent) asymptotic expansions as $t \rightarrow 0$ in $|\arg t|<\pi / 2$ (see Corollaries 1.3-1.5 below). If one considers, for instance, the logarithm of the pairing $\left(q^{\alpha} ; q\right)_{\infty}\left(q^{1-\alpha} ; q\right)_{\infty}$ with $0<\alpha<1$, each term (with $k \geq$ 2 ) in its asymptotic series vanishes again by (3.6) and (3.7). From (2.5), Theorem 1, and Lemma 1 in Section 7 we can in fact prove

Corollary 1.3. For any $0<\alpha, \mu<1$ :

$$
\begin{aligned}
\log \left\{\left(q^{\alpha} ; q\right)_{\infty}\left(q^{1-\alpha} ; q\right)_{\infty}\right\}= & -\frac{\pi^{2}}{3 t}+\log (2 \sin \pi \alpha)+\frac{1}{2} B_{2}(\alpha) t \\
& -\sum_{l=1}^{\infty} l^{-1} \frac{e((1-\alpha) l) \widehat{q}^{l}}{1-\widehat{q}^{l}}-\sum_{l=1}^{\infty} l^{-1} \frac{e(\alpha l) \widehat{q}^{l}}{1-\widehat{q}^{l}}
\end{aligned}
$$

or in exponential form

$$
\begin{aligned}
\left(q^{\alpha} ; q\right)_{\infty}\left(q^{1-\alpha} ; q\right)_{\infty}= & 2(\sin \pi \alpha) \exp \left\{-\frac{\pi^{2}}{3 t}+\frac{1}{2} B_{2}(\alpha) t\right\} \\
& \times(e(1-\alpha) \widehat{q} ; \widehat{q})_{\infty}(e(\alpha) \widehat{q} ; \widehat{q})_{\infty} ; \\
\log \left\{\left(e(\mu) q^{\alpha} ; q\right)_{\infty}\left(e(1-\mu) q^{1-\alpha} ; q\right)_{\infty}\right\} & \\
=- & \left\{\zeta_{\mu}(2)+\zeta_{1-\mu}(2)\right\} t^{-1}-2 \pi i B_{1}(\alpha) B_{1}(\mu)+\frac{1}{2} B_{2}(\alpha) t \\
- & \sum_{l=1}^{\infty} l^{-1} \frac{e((1-\alpha) l) \widehat{q}^{\mu l}}{1-\widehat{q}^{l}}-\sum_{l=1}^{\infty} l^{-1} \frac{e(\alpha l) \widehat{q}^{(1-\mu) l}}{1-\widehat{q}^{l}}
\end{aligned}
$$

or in exponential form

$$
\begin{aligned}
\left(e(\mu) q^{\alpha} ; q\right)_{\infty}\left(e(1-\mu) q^{1-\alpha} ; q\right)_{\infty} & \\
= & \exp \left\{-\left(\zeta_{\mu}(2)+\zeta_{1-\mu}(2)\right) t^{-1}-2 \pi i B_{1}(\alpha) B_{1}(\mu)+\frac{1}{2} B_{2}(\alpha) t\right\} \\
& \times\left(e(1-\alpha) \widehat{q}^{\mu} ; \widehat{q}\right)_{\infty}\left(e(\alpha) \widehat{q}^{1-\mu} ; \widehat{q}\right)_{\infty} .
\end{aligned}
$$

We can now restate Ramanujan's asymptotic formulae (1.6)-(1.8) with explicit error terms. It is known that $F(q)=1 /(q ; q)_{\infty}$ (cf. [Wa, pp. 57-58]), and the famous Rogers-Ramanujan identities assert that

$$
G(q)=\frac{1}{\left(q ; q^{5}\right)_{\infty}\left(q^{4} ; q^{5}\right)_{\infty}} \quad \text { and } \quad H(q)=\frac{1}{\left(q^{2} ; q^{5}\right)_{\infty}\left(q^{3} ; q^{5}\right)_{\infty}}
$$

(cf. [An, (7.1.6) and (7.1.7)]). Formulae (3.8) and (3.10) therefore imply 
COROLlary 1.4. The following formulae hold for $F(q), G(q)$ and $H(q)$ defined by (1.4) and (1.5):

$$
F(q)=\left(\frac{t}{2 \pi}\right)^{1 / 2} \exp \left(\frac{\pi^{2}}{6 t}-\frac{t}{24}\right) \frac{1}{(\widehat{q} ; \widehat{q})_{\infty}},
$$

or in logarithmic form

$$
\begin{aligned}
\log F(q)= & \frac{\pi^{2}}{6 t}+\frac{1}{2} \log \frac{t}{2 \pi}-\frac{t}{24}+\sum_{l=1}^{\infty} l^{-1} \frac{\widehat{q}^{l}}{1-\widehat{q}^{l}} ; \\
G(q)= & \left(\frac{2}{5-\sqrt{5}}\right)^{1 / 2} \exp \left(\frac{\pi^{2}}{15 t}-\frac{t}{60}\right) \\
& \times \frac{1}{\left(e(1 / 5) \widehat{q}^{1 / 5} ; \widehat{q}^{1 / 5}\right)_{\infty}\left(e(4 / 5) \widehat{q}^{1 / 5} ; \widehat{q}^{1 / 5}\right)_{\infty}},
\end{aligned}
$$

or in logarithmic form

$$
\begin{aligned}
\log G(q)= & \frac{\pi^{2}}{15 t}+\frac{1}{2} \log \frac{2}{5-\sqrt{5}}-\frac{t}{60} \\
& +\sum_{l=1}^{\infty} l^{-1} \frac{e(l / 5) \widehat{q}^{l / 5}}{1-\widehat{q}^{l / 5}}+\sum_{l=1}^{\infty} l^{-1} \frac{e(4 l / 5) \widehat{q}^{l / 5}}{1-\widehat{q}^{l / 5}} ; \\
H(q)= & \left(\frac{2}{5+\sqrt{5}}\right)^{1 / 2} \exp \left(\frac{\pi^{2}}{15 t}+\frac{11 t}{60}\right) \\
& \times \frac{1}{\left(e(2 / 5) \widehat{q}^{1 / 5} ; \widehat{q}^{1 / 5}\right)_{\infty}\left(e(3 / 5) \widehat{q}^{1 / 5} ; \widehat{q}^{1 / 5}\right)_{\infty}},
\end{aligned}
$$

or in logarithmic form

$$
\begin{aligned}
\log H(q)= & \frac{\pi^{2}}{15 t}+\frac{1}{2} \log \frac{2}{5+\sqrt{5}}+\frac{11 t}{60} \\
& +\sum_{l=1}^{\infty} l^{-1} \frac{e(2 l / 5) \widehat{q}^{l / 5}}{1-\widehat{q}^{l / 5}}+\sum_{l=1}^{\infty} l^{-1} \frac{e(3 l / 5) \widehat{q}^{l / 5}}{1-\widehat{q}^{l / 5}}
\end{aligned}
$$

In Chapter 16 of his celebrated notebook [Ra1], Ramanujan introduced the symmetric theta function

$$
f(a, b)=\sum_{n=-\infty}^{\infty} a^{n(n+1) / 2} b^{n(n-1) / 2}, \quad|a b|<1,
$$

and studied its various interesting properties; this is fundamental in Ramanujan's theory of theta functions (see [Be4, Chap. 16]). Let $\alpha, \beta, \gamma$ and $\delta$ be positive numbers such that $\alpha+\beta=\gamma+\delta=\omega$, say. He derived in particular the asymptotic result 


$$
\frac{f\left(-q^{\alpha},-q^{\beta}\right)}{f\left(-q^{\gamma},-q^{\delta}\right)} \sim \frac{\sin (\pi \alpha / \omega)}{\sin (\pi \gamma / \omega)}
$$

as $q \rightarrow 1-0$ (cf. [Be5, Chap. 25, Entry 1]). The Jacobi triple product identity

$$
f(a, b)=(-a ; a b)_{\infty}(-b ; a b)_{\infty}(a b ; a b)_{\infty}
$$

(cf. [Be4, Chap. 16, Entry 19]) yields further asymptotic properties:

Corollary 1.5. Let $\alpha, \beta>0$ and $-1 / 2<\mu<1 / 2$, and set $\omega=\alpha+\beta$. Then:

$$
\begin{aligned}
& f\left(-q^{\alpha},-q^{\beta}\right)= 2 \sin (\pi \alpha / \omega) \sqrt{\frac{2 \pi}{\omega t}} \\
& \times \exp \left\{-\frac{\pi^{2}}{2 \omega t}+\frac{1}{2}\left(B_{2}(\alpha / \omega)+\frac{1}{12}\right) \omega t\right\} \\
& \times\left(e(\beta / \omega) \widehat{q}^{1 / \omega} ; \widehat{q}^{1 / \omega}\right)_{\infty}\left(e(\alpha / \omega) \widehat{q}^{1 / \omega} ; \widehat{q}^{1 / \omega}\right)_{\infty}\left(\widehat{q}^{1 / \omega} ; \widehat{q}^{1 / \omega}\right)_{\infty},
\end{aligned}
$$

or in logarithmic form

$\log f\left(-q^{\alpha},-q^{\beta}\right)$

$$
\begin{aligned}
= & -\frac{\pi^{2}}{2 \omega t}-\frac{1}{2} \log \frac{\omega t}{2 \pi}+\log \left(2 \sin \frac{\pi \alpha}{\omega}\right)+\frac{1}{2}\left(B_{2}(\alpha / \omega)+\frac{1}{12}\right) \omega t \\
& -\sum_{l=1}^{\infty} l^{-1} \frac{e(\beta l / \omega) \widehat{q}^{l / \omega}}{1-\widehat{q}^{l / \omega}}-\sum_{l=1}^{\infty} l^{-1} \frac{e(\alpha l / \omega) \widehat{q}^{l / \omega}}{1-\widehat{q}^{l / \omega}}-\sum_{l=1}^{\infty} l^{-1} \frac{\widehat{q}^{l / \omega}}{1-\widehat{q}^{l / \omega}}
\end{aligned}
$$

(3.18) $\quad f\left(e(\mu) q^{\alpha}, e(1-\mu) q^{\beta}\right)$

$$
\begin{aligned}
&= \sqrt{\frac{2 \pi}{\omega t}} \exp \left\{-\left(\zeta_{1 / 2+\mu}(2)+\zeta_{1 / 2-\mu}(2)+\frac{\pi^{2}}{6}\right)(\omega t)^{-1}\right. \\
&\left.-2 \pi i B_{1}(\alpha / \omega) B_{1}(1 / 2+\mu)+\frac{1}{2}\left(B_{2}(\alpha / \omega)+\frac{1}{12}\right) \omega t\right\} \\
& \times\left(e(\beta / \omega) \widehat{q}^{(1 / 2+\mu) / \omega} ; \widehat{q}^{1 / \omega}\right)_{\infty}\left(e(\alpha / \omega) \widehat{q}^{(1 / 2-\mu) / \omega} ; \widehat{q}^{1 / \omega}\right)_{\infty}\left(\widehat{q}^{1 / \omega} ; \widehat{q}^{1 / \omega}\right)_{\infty},
\end{aligned}
$$

or in logarithmic form

$$
\begin{aligned}
\log f\left(e(\mu) q^{\alpha},\right. & \left.e(1-\mu) q^{\beta}\right)=-\left\{\zeta_{1 / 2+\mu}(2)+\zeta_{1 / 2-\mu}(2)+\frac{\pi^{2}}{6}\right\}(\omega t)^{-1} \\
& -\frac{1}{2} \log \frac{\omega t}{2 \pi}-2 \pi i B_{1}(\alpha / \omega) B_{1}(1 / 2+\mu)+\frac{1}{2}\left(B_{2}(\alpha / \omega)+\frac{1}{12}\right) \omega t \\
& -\sum_{l=1}^{\infty} l^{-1} \frac{e(\beta l / \omega) \widehat{q}^{(1 / 2+\mu) l / \omega}}{1-\widehat{q}^{l / \omega}}-\sum_{l=1}^{\infty} l^{-1} \frac{e(\alpha l / \omega) \widehat{q}^{(1 / 2-\mu) l / \omega}}{1-\widehat{q}^{l / \omega}} \\
& -\sum_{l=1}^{\infty} l^{-1} \frac{\widehat{q}^{l / \omega}}{1-\widehat{q}^{l / \omega}} .
\end{aligned}
$$


We next mention a slightly different type of implications from Theorem 1. To this end we prepare some terminology. The q-gamma and $q$-beta functions are defined respectively by

$$
\Gamma_{q}(\alpha)=\frac{(q ; q)_{\infty}}{\left(q^{\alpha} ; q\right)_{\infty}}(1-q)^{1-\alpha} \quad \text { and } \quad B_{q}(\alpha, \beta)=\frac{\Gamma_{q}(\alpha) \Gamma_{q}(\beta)}{\Gamma_{q}(\alpha+\beta)},
$$

whose limits as $q \rightarrow 1-0$ are known to be the ordinary gamma function and the beta function $B(\alpha, \beta)=\Gamma(\alpha) \Gamma(\beta) / \Gamma(\alpha+\beta)$, respectively (cf. [GR, 1.10]). The basic hypergeometric function ${ }_{2} \phi_{1}(a, b ; c ; q, z)$ is defined by

$$
{ }_{2} \phi_{1}(a, b ; c ; q, z)=\sum_{n=0}^{\infty} \frac{(a ; q)_{n}(b ; q)_{n}}{(c ; q)_{n}(q ; q)_{n}} z^{n}, \quad|z|<1,
$$

for any complex $a, b$ and $c$ with $c \neq q^{-n}(n=0,1,2, \ldots)$; the particular case $a=q^{\alpha}, b=q^{\beta}$ and $c=q^{\gamma}$ gives a $q$-analogue of Gauss' hypergeometric function ${ }_{2} F_{1}(\alpha, \beta ; \gamma ; z)$ (cf. [GR, 1.2]). It is known that the classical Gauss and Kummer summation formulae

$$
{ }_{2} F_{1}(\alpha, \beta ; \gamma ; 1)=\frac{\Gamma(\gamma) \Gamma(\gamma-\alpha-\beta)}{\Gamma(\gamma-\alpha) \Gamma(\gamma-\beta)},
$$

where $\operatorname{Re}(\gamma-\alpha-\beta)>0, \gamma \neq-n(n=0,1,2, \ldots)$, and

$$
{ }_{2} F_{1}(\alpha, \beta ; 1+\alpha-\beta ;-1)=\frac{\Gamma(1+\alpha-\beta) \Gamma(1+\alpha / 2)}{\Gamma(1+\alpha) \Gamma(1+\alpha / 2-\beta)},
$$

where $1+\alpha-\beta \neq-n(n=0,1,2, \ldots)$, have $q$-analogues of the form

$$
\begin{array}{r}
{ }_{2} \phi_{1}\left(q^{\alpha}, q^{\beta} ; q^{\gamma} ; q, q^{\gamma-\alpha-\beta}\right)=\frac{\left(q^{\gamma-\alpha} ; q\right)_{\infty}\left(q^{\gamma-\beta} ; q\right)_{\infty}}{\left(q^{\gamma} ; q\right)_{\infty}\left(q^{\gamma-\alpha-\beta} ; q\right)_{\infty}}, \\
{ }_{2} \phi_{1}\left(q^{\alpha}, q^{\beta} ; q^{1+\alpha-\beta} ; q,-q^{1-\beta}\right) \\
=\frac{(-q ; q)_{\infty}\left(q^{1+\alpha} ; q^{2}\right)_{\infty}\left(q^{2+\alpha-2 \beta} ; q^{2}\right)_{\infty}}{\left(q^{1+\alpha-\beta} ; q\right)_{\infty}\left(-q^{1-\beta} ; q\right)_{\infty}}
\end{array}
$$

respectively (cf. [GR, 1.5 and 1.8]). Combining formulae (3.2) and (3.4) with appropriate exponents (in place of $\alpha$ ) we can prove

COROLlary 1.6. Let $\alpha, \beta, \gamma$ be positive numbers. Then the following asymptotic formulae hold for any integer $K \geq 1$ when $t \rightarrow 0$ through $|\arg t| \leq$ $\pi / 2-\delta$ with any small $\delta>0$ :

$$
\begin{aligned}
\log \Gamma_{q}(\alpha)= & \log \Gamma(\alpha)-\frac{1}{4}(\alpha-1)(\alpha-2) t \\
& +\sum_{k=2}^{K-1} \frac{B_{k}}{k k !}\left\{\frac{(-1)^{k} B_{k+1}(\alpha)}{k+1}+1-\alpha\right\} t^{k}+O\left(|t|^{K}\right)
\end{aligned}
$$

for $\alpha>0$; 
(3.26) $\log B_{q}(\alpha, \beta)=\log B(\alpha, \beta)+\frac{1}{2}(\alpha \beta-1) t$

$$
+\sum_{k=2}^{K-1} \frac{B_{k}}{k k !}\left\{\frac{(-1)^{k} C_{k+1}(\alpha, \beta)}{k+1}+1\right\} t^{k}+O\left(|t|^{K}\right)
$$

for $\alpha, \beta>0$, where

$$
C_{k}(\alpha, \beta)=B_{k}(\alpha)+B_{k}(\beta)-B_{k}(\alpha+\beta)
$$

$$
\begin{aligned}
& \log _{2} \phi_{1}\left(q^{\alpha}, q^{\beta} ; q^{\gamma} ; q, q^{\gamma-\alpha-\beta}\right) \\
= & \log _{2} F_{1}(\alpha, \beta ; \gamma ; 1)-\frac{1}{2} \alpha \beta t-\sum_{k=2}^{K-1} \frac{(-1)^{k} B_{k} D_{k+1}(\alpha, \beta, \gamma)}{k(k+1) !} t^{k}+O\left(|t|^{K}\right)
\end{aligned}
$$

for $\gamma-\alpha>0, \gamma-\beta>0, \gamma>0$ and $\gamma-\alpha-\beta>0$, where

$$
D_{k}(\alpha, \beta, \gamma)=B_{k}(\gamma-\alpha)+B_{k}(\gamma-\beta)-B_{k}(\gamma)-B_{k}(\gamma-\alpha-\beta) ;
$$

$$
\begin{aligned}
& \log _{2} \phi_{1}\left(q^{\alpha}, q^{\beta} ; q^{1+\alpha-\beta} ; q,-q^{1-\beta}\right) \\
= & \log _{2} F_{1}(\alpha, \beta ; 1+\alpha-\beta ;-1)-\sum_{k=2}^{K-1} \frac{(-1)^{k} B_{k} E_{k+1}(\alpha, \beta)}{k(k+1) !} t^{k}+O\left(|t|^{K}\right)
\end{aligned}
$$

for $1+\alpha>0,2+\alpha-2 \beta>0,1+\alpha-\beta>0$ and $1-\beta>0$, where

$$
\begin{aligned}
E_{k}(\alpha, \beta)= & 2^{k-1} B_{k}(\alpha / 2+1 / 2)+2^{k-1} B_{k}(1+\alpha / 2-\beta) \\
& -B_{k}(1+\alpha-\beta)-\left(2^{k-1}-1\right) B_{k}(1-\beta) .
\end{aligned}
$$

Here the implied $O$-constants depend at most on $K, \alpha, \beta, \gamma$ and $\delta$.

4. Connections with Ramanujan's formula for $\zeta(2 n+1)$. We next show that our main theorem implies Ramanujan's formula for $\zeta(2 n+1)$ and its several variants. For symmetry of the following results we introduce the new parameter $\tau=t / 2 \pi$. Then the case $\alpha=\beta=1, \lambda=\mu=0$ and $s=2 n+1$ $(n= \pm 1, \pm 2, \ldots)$ of Theorem 0 reduces to the following equivalent form of (1.9).

Theorem 2 (Ramanujan). Let $q=e^{-2 \pi \tau}$ and $\widehat{q}=e^{-2 \pi / \tau}$ with $\operatorname{Re} \tau>0$. Then for any integer $n \neq 0$,

$$
\begin{aligned}
S_{2 n+1}(1,1 ; 0,0 ; q)+\frac{1}{2} \zeta(2 n+1) \\
\quad+\frac{1}{2}(2 \pi)^{2 n+1} \sum_{k=0}^{n+1} \frac{(-1)^{k} B_{2 n+2-2 k} B_{2 k}}{(2 n+2-2 k) !(2 k) !} \tau^{2 n+1-2 k} \\
=(-1)^{n} \tau^{2 n}\left\{S_{2 n+1}(1,1 ; 0,0 ; \widehat{q})+\frac{1}{2} \zeta(2 n+1)\right\} .
\end{aligned}
$$

Theorem 0 further yields the following variants of (1.9). 
TheOREM 3. Let $q$ and $\widehat{q}$ be as in Theorem 2. Then for any integer $n$ and any real $\alpha$ and $\mu$ with $0<\alpha, \mu<1$ :

$$
\begin{aligned}
& S_{2 n+1}(\alpha, 1 ; 0, \mu ; q)+S_{2 n+1}(1-\alpha, 1 ; 0,1-\mu ; q) \\
& \quad+(2 \pi)^{2 n+1} \sum_{k=0}^{2 n+2} \frac{(-i)^{k} B_{2 n+2-k}(\alpha) B_{k}(\mu)}{(2 n+2-k) ! k !} \tau^{2 n+1-k} \\
& =(-1)^{n} \tau^{2 n}\left\{S_{2 n+1}(\mu, 1 ; 0,1-\alpha ; \widehat{q})+S_{2 n+1}(1-\mu, 1 ; 0, \alpha ; \widehat{q})\right\} ;
\end{aligned}
$$

$$
\begin{aligned}
& S_{2 n}(\alpha, 1 ; 0, \mu ; q)-S_{2 n}(1-\alpha, 1 ; 0,1-\mu ; q) \\
& \quad-(2 \pi)^{2 n} \sum_{k=0}^{2 n+1} \frac{(-i)^{k} B_{2 n+1-k}(\alpha) B_{k}(\mu)}{(2 n+1-k) ! k !} \tau^{2 n-k} \\
& =i(-1)^{n} \tau^{2 n-1}\left\{S_{2 n}(\mu, 1 ; 0,1-\alpha ; \widehat{q})-S_{2 n}(1-\mu, 1 ; 0, \alpha ; \widehat{q})\right\},
\end{aligned}
$$

where $B_{k}(x)$ denotes the $k$ th Bernoulli polynomial.

Remark. Eie and Chen [EC, Proposition 3] recently obtained (4.2) in a quite different manner, basing on their theorems for multiple zeta functions associated with polynomials.

TheOREM 4. Let $q$ and $\widehat{q}$ be as in Theorem 2. Then for any integer $n$ and any real $\beta$ and $\lambda$ with $0<\beta, \lambda<1$ :

$$
\begin{aligned}
S_{2 n+1}(1, \beta ; & \lambda, 0 ; q)+S_{2 n+1}(1,1-\beta ; 1-\lambda, 0 ; q)+\zeta(2 n+1, \beta) \\
& +(2 \pi)^{2 n+1} \sum_{k=0}^{2 n+2} \frac{i^{k} \mathcal{B}_{2 n+2-k}(0, e(\lambda)) \mathcal{B}_{k}(0, e(\beta))}{(2 n+2-k) ! k !} \tau^{2 n+1-k} \\
= & (-1)^{n} \tau^{2 n}\left\{S_{2 n+1}(1, \lambda ; 1-\beta, 0 ; \widehat{q})\right. \\
& \left.+S_{2 n+1}(1,1-\lambda ; \beta, 0 ; \widehat{q})+\zeta(2 n+1,1-\lambda)\right\}
\end{aligned}
$$

except when $n=0$;

$$
\begin{aligned}
S_{2 n}(1, \beta ; \lambda, 0 ; q) & -S_{2 n}(1,1-\beta ; 1-\lambda, 0 ; q)+\zeta(2 n, \beta) \\
& -(2 \pi)^{2 n} \sum_{k=0}^{2 n+1} \frac{i^{k} \mathcal{B}_{2 n+1-k}(0, e(\lambda)) \mathcal{B}_{k}(0, e(\beta))}{(2 n+1-k) ! k !} \tau^{2 n-k} \\
= & i(-1)^{n} \tau^{2 n-1}\left\{S_{2 n}(1, \lambda ; 1-\beta, 0 ; \widehat{q})\right. \\
& \left.-S_{2 n}(1,1-\lambda ; \beta, 0 ; \widehat{q})-\zeta(2 n, 1-\lambda)\right\},
\end{aligned}
$$

where $\mathcal{B}_{k}(x, y)$ is defined by $(2.1)$.

5. Asymptotic aspects when $q \rightarrow e(h / k)$. We lastly mention our asymptotic results for $\left(e(\mu) q^{\alpha} ; q\right)_{\infty}$ as $q \rightarrow e(h / k)$, where $h$ and $k$ are relatively prime integers with $k \geq 1$. Let $q_{h / k}=e(h / k) e^{-t}$. 
TheOREM 5. Let $h$ and $k$ be relatively prime integers with $k \geq 1$. Let $\mathcal{B}_{n}(x, y)$ be defined by $(2.1)$, let $\mathcal{C}(\alpha, z)$ denote the constant term of the Laurent series expansion of $\Phi(s, \alpha, z)$ at $s=1$, let $\gamma_{0}=\mathcal{C}_{0}(1,1)$ be the 0 th Euler-Stieltjes constant, and let $\mathcal{D}(\alpha, z)=\Phi^{\prime}(0, \alpha, z)$, where the prime indicates differentiation with respect to $s$. Define

$$
\begin{gathered}
\mathcal{A}_{-1}(\alpha, \mu, h ; k)=k^{-1} \zeta_{\alpha h+\mu k}(2) \\
\mathcal{A}(\alpha, \mu, h ; k)=k^{-1} \sum_{j=1}^{k} e((\alpha h+\mu k) j / k) \\
\times \mathcal{B}_{0}(j / k, e(\alpha h+\mu k)) \mathcal{B}_{1}(\alpha, e(h j / k)) \\
\mathcal{A}_{0}(\alpha, \mu, h ; k)=k^{-1} \sum_{j=1}^{k} e((\alpha h+\mu k) j / k) \mathcal{B}_{0}(j / k, e(\alpha h+\mu k)) \\
\times\left\{\gamma_{0} \mathcal{B}_{1}(\alpha, e(h j / k))+\mathcal{D}(\alpha, e(h j / k))\right\} \\
-\mathcal{B}_{1}(\alpha, e(h j / k)) \mathcal{C}(j / k, e(\alpha h+\mu k)), \\
\mathcal{A}_{n}(\alpha, \mu, h ; k)=k^{-1} \sum_{j=1}^{k} e((\alpha h+\mu k) j / k) \\
\quad \times \mathcal{B}_{n}(j / k, e(\alpha h+\mu k)) \mathcal{B}_{n+1}(\alpha, e(h j / k))
\end{gathered}
$$

for $n=1,2, \ldots$ Then for any real $\alpha$ and $\mu$ with $\alpha>0$, and any integer $N \geq 1$, we have the asymptotic expansion

$$
\begin{aligned}
& \log \left(e(\mu) q_{h / k}^{\alpha} ; q_{h / k}\right)_{\infty} \\
= & -\mathcal{A}_{-1}(\alpha, \mu, h ; k)(k t)^{-1}-\mathcal{A}(\alpha, \mu, h ; k) \log (k t) \\
& -\mathcal{A}_{0}(\alpha, \mu, h ; k)-\sum_{n=1}^{N-1} \frac{(-1)^{n} \mathcal{A}_{n}(\alpha, \mu, h ; k)}{n(n+1) !}(k t)^{n}+O\left\{(k|t|)^{N}\right\}
\end{aligned}
$$

as $t \rightarrow 0$ through the sector $|\arg t| \leq \pi / 2-\delta$ with any small $\delta>0$, where the implied $O$-constant depends at most on $N, h, \alpha, \mu$ and $\delta$.

Let $\psi(z)=\left(\Gamma^{\prime} / \Gamma\right)(z)$ be the digamma function. The case $\alpha=1$ and $\mu=0$ of Theorem 5 reduces to

COROLlary 5.1. Let $h$ and $k$ be relatively prime integers with $k \geq 1$. Then the asymptotic expansion (5.5) holds for $\log \left(q_{h / k} ; q_{h / k}\right)_{\infty}$ with

$$
\begin{aligned}
\mathcal{A}_{-1}(1,0, h ; k) & =\pi^{2} / 6 k \\
\mathcal{A}(1,0, h ; k) & =1 / 2 \\
\mathcal{A}_{0}(1,0, h ; k) & =k^{-1} \sum_{j=1}^{k} \mathcal{B}_{1}(0, e(h j / k)) \psi(j / k)-\log \sqrt{2 \pi k}-\gamma_{0} / 2,
\end{aligned}
$$




$$
\mathcal{A}_{n}(1,0, h ; k)=k^{-1} \sum_{j=1}^{k} B_{n}(j / k) \mathcal{B}_{n+1}(0, e(h j / k))
$$

for $n=1,2, \ldots$

6. Proof of the main theorem. The proof of Theorem 0 begins with the Mellin inversion formula

$$
\frac{q^{\alpha \beta}}{1-e(\lambda) q^{\beta}}=\frac{1}{2 \pi i} \int_{(c)} \Gamma(w) \phi(w, \alpha, \lambda)(\beta t)^{-w} d w
$$

for $|\arg t|<\pi / 2$, where $c$ is a constant satisfying $c>\max (1,1-\operatorname{Re} s)$ and (c) denotes the vertical straight line from $c-i \infty$ to $c+i \infty$; this is obtained from the equality

$$
q^{\alpha \beta}=\frac{1}{2 \pi i} \int_{(c)} \Gamma(w)(\alpha \beta t)^{-w} d w
$$

(cf. [Er2, 6.3, (1)]), by replacing $\alpha$ by $\alpha+l$, multiplying both sides by $e(\lambda l)$, and summing up over $l=0,1, \ldots$ We replace $\beta$ by $\beta+m$ in (6.1), multiply both sides by $e(\alpha \lambda+\beta \mu)(\beta+m)^{-s} e(\mu m)$, and then sum up over $m=0,1, \ldots$ to obtain the formula

$$
\begin{aligned}
& S_{s}(\alpha, \beta ; \lambda, \mu ; q) \\
& \quad=e(\alpha \lambda+\beta \mu) \frac{1}{2 \pi i} \int_{(c)} \Gamma(w) \phi(w, \alpha, \lambda) \phi(s+w, \beta, \mu) t^{-w} d w,
\end{aligned}
$$

which is a key to the following derivation. Here the interchange of the order of summation and integration is justified by the fact that both $w$ and $s+w$ are, by the choice of $c$, in the region of absolute convergence.

We write $w=u+i v$ with real coordinates $u$ and $v$ hereafter, and define

$$
\nu(u ; \alpha, \lambda)=\limsup _{v \rightarrow \pm \infty} \frac{\log |\phi(u+i v, \alpha, \lambda)|}{\log |v|}
$$

for any $u$. By a standard convexity argument, the upper bounds

$$
\nu(u ; \alpha, \lambda)= \begin{cases}1 / 2-u & \text { if } u \leq 0 \\ (1-u) / 2 & \text { if } 0 \leq u \leq 1, \\ 0 & \text { if } u \geq 1\end{cases}
$$

have been proved for any real $\alpha$ and $\lambda$ with $0<\alpha, \lambda \leq 1$ (see [Ka7, Lemma 1]), but the same bounds can also be shown for all positive $\alpha$ and $\lambda$ with a slight extension of the argument. Let $K$ be any nonnegative integer, and let $c_{K}$ be a constant satisfying $-K<c_{K}<-K+1$. Suppose now temporarily that $\operatorname{Re} s<0$. We can then move the path of integration in (6.3) to the left, from $(c)$ to $\left(c_{K}\right)$, passing over the (possible) poles at $w=1-s, 1$ and $w=-k(k=0,1, \ldots, K-1)$, since the integrand is of order $O\left\{|t|^{-u} e^{-(\pi / 2-|\arg t|)|v|}|v|^{\nu+\varepsilon}\right\}$ as $v \rightarrow \pm \infty$, where $\varepsilon$ is any positive 
number and $\nu=\nu(u ; \alpha, \lambda)+\nu(u+\operatorname{Re} s ; \beta, \mu)$. (Note that $|\arg t| \leq \pi / 2-\delta$ with a small $\delta>0$.) We remark that the duplication of the poles above does not occur because of the assumption $s \neq k(k=0,1, \ldots, K)$ of Theorem 0 . Collecting the residues of the relevant poles, and noting that

$$
\operatorname{Res}_{w=1} \phi(w, \alpha, \lambda)=\mathcal{B}_{0}(\alpha, e(\lambda))
$$

and

$$
\phi(-k, \alpha, \lambda)=-\frac{\mathcal{B}_{k+1}(\alpha, e(\lambda))}{k+1} \quad(k=0,1, \ldots)
$$

(cf. [Ap1, p. 164]), we obtain the first assertion (2.3) of Theorem 0 with

$$
\begin{aligned}
& R_{s, K}(\alpha, \beta ; \lambda, \mu ; q) \\
& \quad=e(\alpha \lambda+\beta \mu) \frac{1}{2 \pi i} \int_{\left(c_{K}\right)} \Gamma(w) \phi(w, \alpha, \lambda) \phi(s+w, \beta, \mu) t^{-w} d w .
\end{aligned}
$$

At this stage the temporary restriction on $s$ above can be relaxed to Re $s<K+1$, since the constant $c_{K}$ can be taken as $-K<c_{K}<\min (-K+1$, $1-\operatorname{Re} s)$ in this new situation. The error estimate (2.4) can be derived by further moving the path of integration in (6.6) from $\left(c_{K}\right)$ to $\left(c_{K+1}\right)$, and this gives

$$
\begin{aligned}
R_{s, K}(\alpha, \beta ; \lambda, \mu ; q)= & e(\alpha \lambda+\beta \mu) \frac{(-1)^{K+1}}{(K+1) !} \mathcal{B}_{K+1}(\alpha, e(\lambda)) \phi(s-K, \beta, \mu) t^{K} \\
& +R_{s, K+1}(\alpha, \beta ; \lambda, \mu ; q) .
\end{aligned}
$$

Here, on the right-hand side, the first term is estimated as $\ll|t|^{K}$ with the implied «-constant depending at most on $s, K, \alpha, \beta, \lambda$ and $\mu$, while the integrand of the second term is of order $O\left\{|t|^{-c_{K+1}} e^{-(\pi / 2-|\arg t|)|v|}|v|^{\nu_{K+1}+\varepsilon}\right\}$ as $v \rightarrow \pm \infty$, where $\nu_{K+1}=\nu\left(c_{K+1} ; \alpha, \lambda\right)+\nu\left(c_{K+1}+\operatorname{Re} s ; \beta, \mu\right)$. The assertion (2.4) therefore follows by the choice of $c_{K+1}$.

We lastly proceed to prove (2.5). When $0<\alpha \leq 1$ and $0 \leq \lambda \leq 1$ the functional equation

$$
\begin{aligned}
\phi(w, \alpha, \lambda)= & \frac{\Gamma(1-w)}{(2 \pi)^{1-w}}\left\{e^{\pi i(1-w) / 2} \sum_{l=0}^{\infty}{ }^{\prime} e(-\alpha(\lambda+l))(\lambda+l)^{w-1}\right. \\
& \left.+e^{\pi i(w-1) / 2} \sum_{l=0}^{\infty}{ }^{\prime} e(\alpha(1-\lambda+l))(1-\lambda+l)^{w-1}\right\}
\end{aligned}
$$

holds for $\operatorname{Re} w<0$, where the primed summation symbols indicate the same omission as in Theorem 0 (cf. [Er1, 1.10, (6) and 1.11, (7)]). Suppose now that $0<\alpha, \beta \leq 1,0 \leq \lambda, \mu \leq 1, K \geq 1$ and $\operatorname{Re} s<K$. Here the constant $c_{K}$ can be taken with $-K<c_{K}<\min (-\operatorname{Re} s,-K+1)$. Substituting the functional equations for $\phi(w, \alpha, \lambda)$ and $\phi(s+w, \beta, \mu)$ into the integrand in (6.6), and then changing the order of summation and integration, we find that the integral on the right-hand side (without the factor $e(\alpha \lambda+\beta \mu)$ ) 
becomes

$$
\begin{aligned}
& (2 \pi)^{s-2}\left\{\sum_{l, m=0}^{\infty}{ }^{\prime} e(-\alpha(\lambda+l)-\beta(\mu+m))(\lambda+l)^{-1}(\mu+m)^{s-1}\right. \\
& \times e^{\pi i(2-s) / 2} \tilde{f}_{s, K}\left(4 \pi^{2} e^{-\pi i}(\lambda+l)(\mu+m) / t\right) \\
& +\sum_{l, m=0}^{\infty} e(\alpha(1-\lambda+l)+\beta(1-\mu+m))(1-\lambda+l)^{-1}(1-\mu+m)^{s-1} \\
& \times e^{\pi i(s-2) / 2} \widetilde{f}_{s, K}\left(4 \pi^{2} e^{\pi i}(1-\lambda+l)(1-\mu+m) / t\right) \\
& +\sum_{l, m=0}^{\infty} e(-\alpha(\lambda+l)+\beta(1-\mu+m))(\lambda+l)^{-1}(1-\mu+m)^{s-1} \\
& \times e^{\pi i s / 2} \widetilde{f}_{s, K}\left(4 \pi^{2}(\lambda+l)(1-\mu+m) / t\right) \\
& +\sum_{l, m=0}^{\infty} e(\alpha(1-\lambda+l)-\beta(\mu+m))(1-\lambda+l)^{-1}(\mu+m)^{s-1} \\
& \left.\times e^{-\pi i s / 2} \widetilde{f}_{s, K}\left(4 \pi^{2}(1-\lambda+l)(\mu+m) / t\right)\right\},
\end{aligned}
$$

where

$$
\widetilde{f}_{s, K}(z)=\frac{1}{2 \pi i} \int_{\left(c_{K}\right)} \Gamma(w) \Gamma(1-w) \Gamma(1-s-w) z^{w} d w
$$

for $|\arg z|<3 \pi / 2$. Here the inversion of the order of summation and integration is justified by the fact that both $w$ and $s+w$ are, by the choice of $c_{K}$ above, in the region of absolute convergence. It therefore remains to show that $\widetilde{f}_{s, K}(z)$ can be expressed in terms of the function (2.2). The integral in (6.9) is transformed, upon replacing the variable $w$ by $1-s+w$ (with a slight modification), to

$$
\frac{(-1)^{K} z^{1-s}}{2 \pi i} \int_{\left(d_{K}\right)} \Gamma(K+1-s+w) \Gamma(s-K-w) \Gamma(-w) z^{w} d w
$$

where $d_{K}=c_{K}+\operatorname{Re} s-1$, and hence from the Mellin-Barnes formula for $\Psi(a, c ; z)([\operatorname{Er} 1,6.5,(5)])$, noting that $\operatorname{Re} s-K-1<d_{K}<\min (-1, \operatorname{Re} s-K)$, we obtain

$$
\widetilde{f}_{s, K}(z)=(-1)^{K} z^{1-s} \Gamma(K+1-s) \Psi(K+1-s, K+1-s ; z) .
$$

The assertion (2.5) with (2.6) thus follows from (6.6), (6.8) and (6.10). The proof of Theorem 0 is complete.

7. Proof of Theorem 1 and its corollaries. The aim of this section is to deduce Theorem 1 and its corollaries from Theorem 0 . 
Before starting the proofs we prepare some necessary properties of $\mathcal{B}_{k}(x, y)$ defined by $(2.1)$.

LEMma 1. The following relations hold for any integer $k \geq 0$, and any complex $x$ and $y$ with $y \neq 0$ :

$$
\begin{gathered}
\mathcal{B}_{k}(1-x, 1 / y)=(-1)^{k} y \mathcal{B}_{k}(x, y) \\
\mathcal{B}_{k}(0,1 / y)= \begin{cases}-\mathcal{B}_{1}(0, y)-1 & \text { if } k=1, \\
(-1)^{k} \mathcal{B}_{k}(0, y) & \text { otherwise. }\end{cases}
\end{gathered}
$$

Proof. Equating the coefficients of the Taylor series (near $z=0$ ) for both sides of the identities

$$
\frac{z e^{(1-x) z}}{e^{z} / y-1}=\frac{-y z e^{-x z}}{y e^{-z}-1}, \quad \frac{z}{e^{z} / y-1}=\frac{-z}{y e^{-z}-1}-z,
$$

we obtain (7.1) and (7.2) respectively.

Proof of Theorem 1, Corollaries 1.1 and 1.2. Let $K \geq 1$ and $0 \leq \mu \leq 1$ in Theorem 0 . Then in the region $\operatorname{Re} s<K+1$ except at $s=k(k=$ $0,1, \ldots, K)$,

$$
\begin{aligned}
S_{s}(\alpha, 1 ; 0, \mu ; q)= & e(\mu) \mathcal{B}_{0}(1, e(\mu)) \Gamma(1-s) \zeta(1-s, \alpha) t^{s-1} \\
& +\zeta_{\mu}(s+1) t^{-1}-B_{1}(\alpha) \zeta_{\mu}(s) \\
& +\sum_{k=1}^{K-1} \frac{(-1)^{k+1} B_{k+1}(\alpha)}{(k+1) !} \zeta_{\mu}(s-k) t^{k} \\
& +R_{s, K}(\alpha, 1 ; 0, \mu ; q) .
\end{aligned}
$$

We may let $s \rightarrow 1$ in this formula, since $S_{s}$ and $R_{s, K}$ are both holomorphic at least in $\operatorname{Re} s<K+1$.

Consider first the case of $\mu=0$ or 1 . Setting $s=1+\varepsilon$, and noting that, as $\varepsilon \rightarrow 0$,

$$
\begin{aligned}
\Gamma(-\varepsilon) & =-\varepsilon^{-1}-\gamma_{0}+O(\varepsilon), \\
\zeta(-\varepsilon, \alpha) & =\zeta(0, \alpha)-\zeta^{\prime}(0, \alpha) \varepsilon+O\left(\varepsilon^{2}\right), \\
\zeta(1+\varepsilon) & =\varepsilon^{-1}+\gamma_{0}+O(\varepsilon),
\end{aligned}
$$

with $\zeta(0, \alpha)=-B_{1}(\alpha)$ and $\zeta^{\prime}(0, \alpha)=\log \{\Gamma(\alpha) / \sqrt{2 \pi}\}$ (cf. [Er1, 1.10, (10) and $(11)])$, we see that the sum of the first three terms on the right-hand side of (7.3) tends, as $s \rightarrow 1$, to

$$
\zeta(2) t^{-1}+B_{1}(\alpha) \log t+\log \frac{\Gamma(\alpha)}{\sqrt{2 \pi}},
$$

and hence (3.2) follows from the fact that

$$
\zeta(-k)= \begin{cases}-1 / 2 & \text { if } k=0, \\ -B_{k+1} /(k+1) & \text { if } k=1,2, \ldots\end{cases}
$$

(cf. [Er1, 1.12, (18) and (20)]). 
Next in the case of $0<\mu<1$ the first term on the right-hand side of (7.3) vanishes by (2.7), and hence (3.3) is derived from (2.8) and the following lemma.

Lemma 2. For any real $\mu$ with $0<\mu<1$,

$$
\begin{aligned}
\zeta_{\mu}(1) & =-\log (2 \sin \pi \mu)-\pi i B_{1}(\mu), \\
\zeta_{\mu}(-k) & = \begin{cases}-\mathcal{B}_{1}(0, e(\mu))-1 & \text { if } k=0, \\
-\mathcal{B}_{k+1}(0, e(\mu)) /(k+1) & \text { if } k=1,2, \ldots\end{cases}
\end{aligned}
$$

Proof. From the series representation we have

$$
\zeta_{\mu}(1)=-\log (1-e(\mu))=-\log |1-e(\mu)|-i \arg (1-e(\mu))
$$

with the principal branch of logarithms, and hence (7.5) follows. Next noting that

$$
\begin{aligned}
\zeta_{\mu}(-k) & =e(\mu) \phi(\mu, 1,-k)=-\frac{e(\mu) \mathcal{B}_{k+1}(1, e(\mu))}{k+1} \\
& =\frac{(-1)^{k} \mathcal{B}_{k+1}(0, e(-\mu))}{k+1} \quad(k=0,1, \ldots)
\end{aligned}
$$

by (6.5) and (7.1), and further using (7.2), we obtain (7.6).

REMARK. Equality (7.6) is in fact valid for any real $\mu$, since $\mathcal{B}_{k}(0,1)$ $=B_{k}$ for all $k \geq 0$ (see $\left.(7.4)\right)$.

Corollary 1.1 readily follows from (3.3) by noting that

$$
\zeta_{1 / 2}(2)=\sum_{n=1}^{\infty}(-1)^{n} n^{-2}=\left(2^{-1}-1\right) \zeta(2), \quad \mathcal{B}_{k}(0,-1)=\left(2^{k}-1\right) B_{k}
$$

for any integer $k \geq 0$, where the last equality is obtained by equating the coefficients of the Taylor series (near $z=0$ ) for both sides of the identity

$$
\frac{z}{-e^{z}-1}=\frac{2 z}{e^{2 z}-1}-\frac{z}{e^{z}-1} .
$$

Corollary 1.2 can be derived from (3.2), (3.4) and the fact that $B_{k} B_{k+1}(1)$ $=0$ for any $k \geq 2$ (see (3.6) and (3.7)), where the explicit evaluations of the remainder terms are given in the case $n=0$ of Lemma 4 below (see also $(1.3))$.

Proof of Corollaries 1.3-1.5. We first prove Corollary 1.3. It can be observed that the terms with $2 \leq k \leq K-1$ in the asymptotic series for $\log \left\{\left(e(\mu) q^{\alpha} ; q\right)_{\infty}\left(e(1-\mu) q^{1-\alpha} ; q\right)_{\infty}\right\}$ vanish by (3.6) and (3.7) if $\mu=0$ or 1 , and by (3.7) and (7.2) if $0<\mu<1$. Formulae (3.10) and (3.11) therefore follow from the reciprocal formula for the gamma function (cf. [Er1, 1.2, (6)]) and the explicit evaluation of the pairing of the remainder terms, which is given in the case $n=0$ of (8.7) and (8.8) below (see also (1.3)).

We next prove Corollary 1.4. Formula (3.12) is immediate from (3.8). We replace $t$ by $5 t$ in (3.10). Formulae (3.13) and (3.14) are then obtained 
by setting $\alpha=1 / 5$ and $\alpha=2 / 5$ respectively, since $\widehat{q^{5}}=\widehat{q}^{1 / 5}$ by $(3.5)$, upon noting that

$$
\sin \frac{\pi}{5}=\frac{1}{2} \sqrt{\frac{5-\sqrt{5}}{2}} \text { and } \sin \frac{2 \pi}{5}=\frac{1}{2} \sqrt{\frac{5+\sqrt{5}}{2}} .
$$

We lastly prove Corollary 1.5. It follows from (3.16) that

$$
f\left(-q^{\alpha},-q^{\beta}\right)=\left(q^{\alpha} ; q^{\omega}\right)_{\infty}\left(q^{\beta} ; q^{\omega}\right)_{\infty}\left(q^{\omega} ; q^{\omega}\right)_{\infty}
$$

and

$$
f\left(e(\mu) q^{\alpha}, e(1-\mu) q^{\beta}\right)=\left(e(1 / 2+\mu) q^{\alpha} ; q^{\omega}\right)_{\infty}\left(e(1 / 2-\mu) q^{\beta} ; q^{\omega}\right)_{\infty}\left(q^{\omega} ; q^{\omega}\right)_{\infty}
$$
with $-1 / 2<\mu<1 / 2$. We replace $t$ by $\omega t, \alpha$ by $\alpha / \omega$, and $1-\alpha$ by $1-\alpha / \omega=$ $\beta / \omega$ in (3.8), (3.10) and (3.11), and further $\mu$ by $1 / 2+\mu$ in (3.11). Since $q^{\omega}=\widehat{q}^{1 / \omega}$ by $(3.5)$, formula $(3.17)$ is therefore obtained by combining the resulting (3.8) with (3.10), while (3.18) follows by combining the resulting (3.8) with (3.11) upon noting that $0<1 / 2 \pm \mu<1$.

Proof of Corollary 1.6. Truncation of the Taylor series

$$
\log \left(\frac{1-e^{-t}}{t}\right)=\sum_{k=1}^{\infty} \frac{B_{k}}{k k !} t^{k}, \quad|t|<2 \pi
$$

gives the asymptotic expansion

$$
\log (1-q)=\log t+\sum_{k=1}^{K-1} \frac{B_{k}}{k k !} t^{k}+O\left(|t|^{K}\right)
$$

for any integer $K \geq 1$, as $t \rightarrow 0$ in the sector $|\arg t| \leq \pi-\delta$ with any small $\delta>0$, where the implied $O$-constant depends only on $K$ and $\delta$. Formula (3.25) is therefore obtained by combining (3.2), (3.8) with (7.7), where the last infinite sum in (3.8) is absorbed into the resulting error term $O\left(|t|^{K}\right)$ (see Remark 2 of Corollary 1.2). Formula (3.26) then readily follows from the definition of $B_{q}(\alpha, \beta)$ and (3.25). Formula (3.27) is derived from (3.23) by using (3.2) (with appropriate exponents), where the coefficient of $t^{0}$ is evaluated by (3.21). Formula (3.28) follows from (3.24) by using (3.2), (3.4) (with appropriate exponents) and (3.9), where the coefficient of $t^{0}$ is evaluated by (3.22) and the duplication formula for the gamma function (cf. [Er1, $1.2,(15)])$.

8. Proofs of Theorems 2, 3 and 4. Before proceeding to the proofs we state

Lemma 3. Let $f_{s, K}(z)$ be defined by (2.6). Then for any integer $K \geq 0$, any $z$ with $|\arg z|<\pi / 2$, and $\operatorname{Re} s<K+1$,

$$
e^{\pi i s} f_{s, K}\left(e^{-\pi i} z\right)-e^{-\pi i s} f_{s, K}\left(e^{\pi i} z\right)=\frac{2 \pi i(-1)^{K}}{\Gamma(K+1-s)} e^{-z} .
$$


Proof. The Mellin-Barnes formula for $\Psi(a, c ; z)$ (cf. [Er1, 6.5, (5)]) implies, by the reciprocal formula for the gamma function (cf. [Er1, 1.2, (6)]), that

$$
f_{s, K}(z)=\frac{(-1)^{K}}{2 i} \int_{(b)} \frac{\Gamma(-w)}{\Gamma(K+1-s) \sin (\pi(s-w))} z^{w} d w
$$

for $|\arg z|<3 \pi / 2$ and $\operatorname{Re} s<K+1$, where $b$ is a constant satisfying $\operatorname{Re} s-K-1<b<0$. We therefore find that the left-hand side of (8.1) equals

$$
(-1)^{K} \int_{(b)} \frac{\Gamma(-w)}{\Gamma(K+1-s)} z^{w} d w
$$

which is further transformed to the right-hand side of (8.1) by the Mellin inversion formula for the exponential function (cf. [Er2, 6.3, (1)]).

We first deduce Theorem 2 from Theorem 0 . Let $n$ and $K$ be integers with $n \neq 0$ and $K \geq \max (2 n+2,1)$, and let $\operatorname{Re} s<K$. The case $\alpha=\beta=1$ and $\lambda=\mu=0$ of (2.3) reduces, by (3.7), to

$$
\begin{aligned}
S_{s}(1,1 ; 0,0 ; q)= & \Gamma(1-s) \zeta(1-s) t^{s-1}+\sum_{\substack{k=-1 \\
k \neq 0,2 n}}^{K-1} \frac{B_{k+1}}{(k+1) !} \zeta(s-k) t^{k} \\
& +B_{1} \zeta(s)+R_{s, K}(1,1 ; 0,0 ; q),
\end{aligned}
$$

where the exclusion of the term with $k=2 n$ on the right-hand side is justified by (3.6) and $n \neq 0$. We may let $s \rightarrow 2 n+1$ in this equality, since $S_{s}$ and $R_{s, K}$ are both holomorphic at least in $\operatorname{Re} s<K$. The first term on the right-hand side of (8.2) is equal to $\zeta(s) \tau^{s-1} / 2 \sin (\pi s / 2)$ by the functional equation of $\zeta(s)$, and this shows that the right-hand side of (8.2) tends, as $s \rightarrow 2 n+1$, to

$$
\begin{aligned}
& \frac{1}{2}(-1)^{n} \zeta(2 n+1) \tau^{2 n}+\sum_{\substack{k=-1 \\
k \neq 0,2 n}}^{2 n+1} \frac{B_{k+1}}{(k+1) !} \zeta(2 n+1-k) t^{k} \\
&+B_{1} \zeta(2 n+1)+R_{2 n+1, K}(1,1 ; 0,0 ; q),
\end{aligned}
$$

where the terms with $2 n+2 \leq k \leq K-1$ vanish by (3.6) and (7.4). The assertion (4.1) therefore follows from (3.6) and the fact that $\zeta(2 h)=$ $(-1)^{h+1}(2 \pi)^{2 h} B_{2 h} / 2(2 h)$ ! for $h=0,1, \ldots$ (cf. [Er1, 1.12, (18) and (21)]), where the explicit evaluation of the remainder term is given by (8.3) below.

Lemma 4. For any integers $n$ and $K$ with $K \geq \max (2 n+2,1)$,

$$
\begin{aligned}
R_{2 n+1, K}(1,1 ; 0,0 ; q) & =(-1)^{n} \tau^{2 n} S_{2 n+1}(1,1 ; 0,0 ; \widehat{q}), \\
R_{2 n+1, K}(1,1 ; 0,1 / 2 ; q) & =(-1)^{n} \tau^{2 n} S_{2 n+1}(1 / 2,1 ; 0,0 ; \widehat{q}) .
\end{aligned}
$$


Proof. The abbreviation $f(z)=f_{s, K}(z)$ is used throughout. We consider the case $s=2 n+1, \alpha=\beta=1$ and $\lambda=\mu=0$ in (2.5). It is seen that the third and fourth infinite sums on the right-hand side cancel each other, because of the factors $e^{ \pm \pi i s / 2}$, and hence

$$
\begin{aligned}
R_{2 n+1, K}(1,1 ; 0,0 ; q) & \\
= & (-1)^{K}(2 \pi)^{-2 n-1} t^{2 n} \Gamma(K-2 n) \cdot(-1)^{n} i \sum_{l, m=0}^{\infty}(1+l)^{-2 n-1} \\
& \times\left\{f\left(4 \pi^{2} e^{-\pi i}(1+l)(1+m) / t\right)-f\left(4 \pi^{2} e^{\pi i}(1+l)(1+m) / t\right)\right\} \\
= & (-1)^{n} \tau^{2 n} \sum_{l, m=0}^{\infty}(1+l)^{-2 n-1} \widehat{q}^{(1+l)(1+m)},
\end{aligned}
$$

by Lemma 3. This implies (8.3).

On the other hand, when $\alpha=\beta=1, \lambda=0$ and $\mu=1 / 2$ in (2.5) the same cancellation as above occurs, and hence

$$
\begin{aligned}
R_{2 n+1, K} & (1,1 ; 0,1 / 2 ; q) \\
= & (-1)^{K}(2 \pi)^{-2 n-1} t^{2 n} \Gamma(K-2 n) \cdot(-1)^{n} i \sum_{l, m=0}^{\infty}(1+l)^{-2 n-1} \\
& \times\left\{f\left(4 \pi^{2} e^{-\pi i}(1+l)(1 / 2+m) / t\right)-f\left(4 \pi^{2} e^{\pi i}(1+l)(1 / 2+m) / t\right)\right\},
\end{aligned}
$$

which implies (8.4), again by Lemma 3 .

We proceed to prove variants of Ramanujan's formula for $\zeta(2 n+1)$.

Proof of Theorem 3. Let $n$ and $K$ be integers with $K \geq \max (2 n+2,1)$, and let $\operatorname{Re} s<K$. Then formula (2.3) with (2.7) and (3.7) gives

$$
\begin{aligned}
& S_{s}(\alpha, 1 ; 0, \mu ; q)+S_{s}(1-\alpha, 1 ; 0,1-\mu ; q) \\
& =\sum_{k=-1}^{K-1} \frac{(-1)^{k+1} B_{k+1}(\alpha)}{(k+1) !}\left\{\zeta_{\mu}(s-k)+(-1)^{k+1} \zeta_{1-\mu}(s-k)\right\} t^{k} \\
& \quad+R_{s, K}(\alpha, 1 ; 0, \mu ; q)+R_{s, K}(1-\alpha, 1 ; 0,1-\mu ; q) .
\end{aligned}
$$

We may let $s \rightarrow 2 n+1$ in this equality, since $S_{s}$ and $R_{s, K}$ are both holomorphic at least in Re $s<K$. In passing to the limit, the factor $\zeta_{\mu}(s-k)+$ $(-1)^{k+1} \zeta_{1-\mu}(s-k)$ on the right-hand side vanishes if $2 n+2 \leq k \leq K-1$ by (7.2) and (7.6), while it tends to $-(2 \pi i)^{2 n+1-k} B_{2 n+1-k}(\mu) /(2 n+1-k)$ ! if $-1 \leq k \leq 2 n+1$ by the following lemma.

Lemma 5. For any integer $h \geq 0$ and any real $\mu$ with $0<\mu<1$,

$$
\zeta_{\mu}(h)+(-1)^{h} \zeta_{1-\mu}(h)=-\frac{(2 \pi i)^{h}}{h !} B_{h}(\mu) .
$$


Proof. From (6.7) we have the functional equation

$$
\zeta(1-s, \mu)=\frac{\Gamma(s)}{(2 \pi)^{s}}\left\{e^{-\pi i s / 2} \zeta_{\mu}(s)+e^{\pi i s / 2} \zeta_{1-\mu}(s)\right\} .
$$

To obtain the assertion for $h \geq 1$, we set $s=h$. The remaining case $h=0$ follows by noting $\zeta_{\mu}(0)=(-1+i \cot \pi \mu) / 2($ see $(2.8)$ and $(7.6))$.

Furthermore the explicit evaluation of the pairing of the remainder terms, when $s \rightarrow 2 n+1$, is given by (8.8) below.

Lemma 6. For any $\alpha$ and $\mu$ with $0<\alpha, \mu<1$

$$
\begin{aligned}
& R_{2 n+1, K}(\alpha, 1 ; 0,0 ; q)+R_{2 n+1, K}(1-\alpha, 1 ; 0,0 ; q) \\
& \quad=(-1)^{n} \tau^{2 n}\left\{S_{2 n+1}(1,1 ; 0,1-\alpha ; \widehat{q})+S_{2 n+1}(1,1 ; 0, \alpha ; \widehat{q})\right\}
\end{aligned}
$$

where $n$ and $K$ are integers with $K \geq \max (2 n+2,1)$;

$$
\begin{aligned}
& R_{2 n+1, K}(\alpha, 1 ; 0, \mu ; q)+R_{2 n+1, K}(1-\alpha, 1 ; 0,1-\mu ; q) \\
& =(-1)^{n} \tau^{2 n}\left\{S_{2 n+1}(\mu, 1 ; 0,1-\alpha ; \widehat{q})+S_{2 n+1}(1-\mu, 1 ; 0, \alpha ; \widehat{q})\right\},
\end{aligned}
$$

where $n$ and $K$ are integers with $K \geq \max (2 n+2,1)$;

$$
\begin{aligned}
& R_{2 n, K}(\alpha, 1 ; 0, \mu ; q)-R_{2 n, K}(1-\alpha, 1 ; 0,1-\mu ; q) \\
& \quad=i(-1)^{n} \tau^{2 n-1}\left\{S_{2 n}(\mu, 1 ; 0,1-\alpha ; \widehat{q})-S_{2 n}(1-\mu, 1 ; 0, \alpha ; \widehat{q})\right\},
\end{aligned}
$$

where $n$ and $K$ are integers with $K \geq \max (2 n+1,1)$.

Proof. We first prove (8.8). We set $s=2 n+1$ in (2.5). Looking at the sum on the left-hand side of (8.8), we observe that the third and fourth infinite sums of $R_{2 n+1, K}(\alpha, 1 ; 0, \mu ; q)$ cancel the fourth and third infinite sums of $R_{2 n+1, K}(1-\alpha, 1 ; 0,1-\mu ; q)$ respectively, because of the factors $e^{ \pm \pi i s / 2}$. The first and second infinite sums of $R_{2 n+1, K}(\alpha, 1 ; 0, \mu ; q)$ are then combined with the second and first infinite sums of $R_{2 n+1, K}(1-\alpha, 1 ; 0,1-\mu ; q)$ respectively, and hence the left-hand side of (8.8) is equal to

$$
\begin{aligned}
(-1)^{K} & (2 \pi)^{-2 n-1} t^{2 n} \Gamma(K-2 n) \cdot i(-1)^{n} \\
& \times\left[\sum_{l, m=0}^{\infty} e(-\alpha(1+l))(1+l)^{-2 n-1}\right. \\
& \times\left\{f\left(4 \pi^{2} e^{-\pi i}(1+l)(\mu+m) / t\right)-f\left(4 \pi^{2} e^{\pi i}(1+l)(\mu+m) / t\right)\right\} \\
& +\sum_{l, m=0}^{\infty} e(\alpha(1+l))(1+l)^{-2 n-1} \\
& \left.\times\left\{f\left(4 \pi^{2} e^{-\pi i}(1+l)(1-\mu+m) / t\right)-f\left(4 \pi^{2} e^{\pi i}(1+l)(1-\mu+m) / t\right)\right\}\right]
\end{aligned}
$$




$$
\begin{aligned}
= & (-1)^{n} \tau^{2 n}\left\{\sum_{l, m=0}^{\infty} e(-\alpha(1+l))(1+l)^{-2 n-1} \widehat{q}^{(1+l)(\mu+m)}\right. \\
& \left.+\sum_{l, m=0}^{\infty} e(\alpha(1+l))(1+l)^{-2 n-1} \widehat{q}^{(1+l)(1-\mu+m)}\right\},
\end{aligned}
$$

by Lemma 3. This implies (8.8). The proof of (8.7) is almost the same.

We next prove (8.9). We set $s=2 n$ in (2.5). The same cancellations and combinations as above occur for the difference on the left-hand side of (8.9), and this shows that the left-hand side is equal to

$$
\begin{aligned}
(-1)^{K} & (2 \pi)^{-2 n} t^{2 n-1} \Gamma(K+1-2 n) \cdot(-1)^{n} \\
& \times\left[\sum_{l, m=0}^{\infty} e(-\alpha(1+l))(1+l)^{-2 n}\right. \\
& \times\left\{f\left(4 \pi^{2} e^{-\pi i}(1+l)(\mu+m) / t\right)-f\left(4 \pi^{2} e^{\pi i}(1+l)(\mu+m) / t\right)\right\} \\
& -\sum_{l, m=0}^{\infty} e(\alpha(1+l))(1+l)^{-2 n} \\
& \left.\times\left\{f\left(4 \pi^{2} e^{-\pi i}(1+l)(1-\mu+m) / t\right)-f\left(4 \pi^{2} e^{\pi i}(1+l)(1-\mu+m) / t\right)\right\}\right]
\end{aligned}
$$

which implies (8.9), again by Lemma 3 .

The derivation of (4.3) is almost the same as that of (4.2) except that we use the equality

$$
\begin{aligned}
& S_{s}(\alpha, 1 ; 0, \mu ; q)-S_{s}(1-\alpha, 1 ; 0,1-\mu ; q) \\
& =\sum_{k=-1}^{K-1} \frac{(-1)^{k+1} B_{k+1}(\alpha)}{(k+1) !}\left\{\zeta_{\mu}(s-k)-(-1)^{k+1} \zeta_{1-\mu}(s-k)\right\} t^{k} \\
& \quad+R_{s, K}(\alpha, 1 ; 0, \mu ; q)-R_{s, K}(1-\alpha, 1 ; 0,1-\mu ; q),
\end{aligned}
$$

and (8.9) instead of (8.5) and (8.8) respectively. The proof of Theorem 3 is complete.

Proof of Theorem 4. We first prove (4.4). Let $n$ and $K$ be integers with $n \neq 0$ and $K \geq \max (2 n+2,1)$, and let $\operatorname{Re} s<K$. From (6.7) we have the functional equation

$$
\zeta_{\lambda}(1-s)=\frac{\Gamma(s)}{(2 \pi)^{s}}\left\{e^{\pi i s / 2} \zeta(s, \lambda)+e^{-\pi i s / 2} \zeta(s, 1-\lambda)\right\} .
$$

From this together with (7.1) and (7.2), formula (2.3) gives 


$$
\begin{aligned}
& S_{s}(1, \beta ; \lambda, 0 ; q)+S_{s}(1,1-\beta ; 1-\lambda, 0 ; q) \\
& =\frac{\tau^{s-1}}{2 \sin (\pi s / 2)}\{\zeta(s, \lambda)+\zeta(s, 1-\lambda)\}+\sum_{k=-1}^{K-1} \frac{(-1)^{k+1} \mathcal{B}_{k+1}(0, e(\lambda))}{(k+1) !} \\
& \quad \times\left\{\zeta(s-k, \beta)+(-1)^{k+1} \zeta(s-k, 1-\beta)\right\} t^{k}+R_{s, K}(1, \beta ; \lambda, 0 ; q) \\
& \quad+R_{s, K}(1,1-\beta ; 1-\lambda, 0 ; q)-\zeta(s, \beta),
\end{aligned}
$$

where the extra $-\zeta(s, \beta)$ on the last line comes from the term with $k=0$ in the finite sum on the right-hand side (see the case $k=1$ of (7.2)). We may let $s \rightarrow 2 n+1$ in this equality, since $S_{s}$ and $R_{s, K}$ are both holomorphic at least in $\operatorname{Re} s<K$. We here restrict ourselves to the case $n \geq 1$. In passing to the limit, the factor $\zeta(s-k, \beta)+(-1)^{k+1} \zeta(s-k, 1-\beta)$ on the right-hand side vanishes if $2 n+2 \leq k \leq K-1$ by (3.7) and (6.5), while it tends to $-(-2 \pi i)^{2 n+1-k} \mathcal{B}_{2 n+1-k}(0, e(\beta)) /(2 n+1-k)$ ! if $-1 \leq k \leq 2 n+1$ except at $k=2 n$, and to $2 \pi i \mathcal{B}_{1}(0, e(\lambda))+\pi i$ if $k=2 n$, by the following lemma.

LEMma 7. For any integer $h \geq 0$ and any real $\beta$ with $0<\beta<1$,

$$
\begin{gathered}
\zeta(h, \beta)+(-1)^{h} \zeta(h, 1-\beta)=-\frac{(-2 \pi i)^{h}}{h !} \mathcal{B}_{h}(0, e(\beta)) \quad \text { if } h \neq 1, \\
\zeta(s, \beta)-\left.\zeta(s, 1-\beta)\right|_{s=1}=2 \pi i \mathcal{B}_{1}(0, e(\beta))+\pi i .
\end{gathered}
$$

Proof. The assertion (8.12) for $h \geq 2$ follows by setting $s=h$ in (8.10) and using (7.6), while (8.13) follows from $\zeta(s, \beta)-\left.\zeta(s, 1-\beta)\right|_{s=1}=$ $\pi \cot \pi \beta$ and (2.8). In the remaining case $h=0$ the left-hand side of (8.12) is 0 by (3.7) and (6.5), and hence the assertion also follows from (2.7) in this case.

The other extra term, caused by the last $\pi i$ in (8.13), appears on the right-hand side of (8.11), and it is further modified as $\frac{(-1)^{2 n+1} t^{2 n}}{(2 n+1) !} \mathcal{B}_{2 n+1}(0, e(\lambda)) \pi i=\frac{(-1)^{n+1}}{2} \tau^{2 n}\{\zeta(2 n+1, \lambda)-\zeta(2 n+1,1-\lambda)\}$, again by (8.12). This is combined with the first term on the right-hand side of (8.11) to imply the assertion (4.4), where the explicit evaluation of the pairing of the remainder terms is given by (8.14) below.

Lemma 8. For any real $\beta$ and $\lambda$ with $0<\beta, \lambda<1$ :

$$
\begin{aligned}
& R_{2 n+1, K}(1, \beta ; \lambda, 0 ; q)+R_{2 n+1, K}(1,1-\beta ; 1-\lambda, 0 ; q) \\
& \quad=(-1)^{n} \tau^{2 n}\left\{S_{2 n+1}(1, \lambda ; 1-\beta, 0 ; \widehat{q})+S_{2 n+1}(1,1-\lambda ; \beta, 0 ; \widehat{q})\right\},
\end{aligned}
$$

where $n$ and $K$ are integers with $K \geq \max (2 n+2,1)$;

$$
\begin{aligned}
& R_{2 n, K}(1, \beta ; \lambda, 0 ; q)-R_{2 n, K}(1,1-\beta ; 1-\lambda, 0 ; q) \\
& \quad=i(-1)^{n} \tau^{2 n-1}\left\{S_{2 n}(1, \lambda ; 1-\beta, 0 ; \widehat{q})-S_{2 n}(1,1-\lambda ; \beta, 0 ; \widehat{q})\right\},
\end{aligned}
$$

where $n$ and $K$ are integers with $K \geq \max (2 n+1,1)$. 
Proof. We first prove (8.14). We may set $s=2 n+1$ in (2.5). In the sum on the left-hand side of (8.14), the same cancellations and combinations occur as in the proof of Lemma 6, and hence the left-hand side is equal to

$$
\begin{aligned}
(-1)^{K} & (2 \pi)^{-2 n-1} t^{2 n} \Gamma(K-2 n) \cdot i(-1)^{n} \\
& \times\left[\sum_{l, m=0}^{\infty} e(-\beta(1+m))(\lambda+l)^{-2 n-1}\right. \\
& \times\left\{f\left(4 \pi^{2} e^{-\pi i}(\lambda+l)(1+m) / t\right)-f\left(4 \pi^{2} e^{\pi i}(\lambda+l)(1+m) / t\right)\right\} \\
& +\sum_{l, m=0}^{\infty} e(\beta(1+m))(1-\lambda+l)^{-2 n-1} \\
& \left.\times\left\{f\left(4 \pi^{2} e^{-\pi i}(1-\lambda+l)(1+m) / t\right)-f\left(4 \pi^{2} e^{\pi i}(1-\lambda+l)(1+m) / t\right)\right\}\right] \\
= & (-1)^{n} \tau^{2 n}\left\{\sum_{l, m=0}^{\infty} e(-\beta(1+m))(\lambda+l)^{-2 n-1} \widehat{q}^{(\lambda+l)(1+m)}\right. \\
& \left.+\sum_{l, m=0}^{\infty} e(\beta(1+m))(1-\lambda+l)^{-2 n-1} \widehat{q}^{(1-\lambda+l)(1+m)}\right\},
\end{aligned}
$$

by Lemma 3. This implies (8.14).

Similarly to the preceding case, it is shown that the left-hand side of (8.15) is equal to

$$
\begin{aligned}
(-1)^{K} & (2 \pi)^{-2 n} t^{2 n-1} \Gamma(K+1-2 n) \cdot(-1)^{n} \\
& \times\left[\sum_{l, m=0}^{\infty} e(-\beta(1+m))(\lambda+l)^{-2 n}\right. \\
& \times\left\{f\left(4 \pi^{2} e^{-\pi i}(\lambda+l)(1+m) / t\right)-f\left(4 \pi^{2} e^{\pi i}(\lambda+l)(1+m) / t\right)\right\} \\
& -\sum_{l, m=0}^{\infty} e(\beta(1+m))(1-\lambda+l)^{-2 n} \\
& \left.\times\left\{f\left(4 \pi^{2} e^{-\pi i}(1-\lambda+l)(1+m) / t\right)-f\left(4 \pi^{2} e^{\pi i}(1-\lambda+l)(1+m) / t\right)\right\}\right]
\end{aligned}
$$

which implies (8.15), again by Lemma 3 .

The remaining case $n \leq-1$ of (4.4) is proved similarly upon noting that $\zeta(2 n+1, \lambda)=\zeta(2 n+1,1-\lambda)$ holds by (3.7) (or (7.1)) and (6.5).

Furthermore, the derivation of (4.5) is almost the same as that of (4.4) except that we use the equality 


$$
\begin{aligned}
& S_{s}(1, \beta ; \lambda, 0 ; q)-S_{s}(1,1-\beta ; 1-\lambda, 0 ; q) \\
&= \frac{i \tau^{s-1}}{2 \cos (\pi s / 2)}\{\zeta(s, \lambda)-\zeta(s, 1-\lambda)\}+\sum_{k=-1}^{K-1} \frac{(-1)^{k+1} \mathcal{B}_{k+1}(0, e(\lambda))}{(k+1) !} \\
& \times\left\{\zeta(s-k, \beta)-(-1)^{k+1} \zeta(s-k, 1-\beta)\right\} t^{k} \\
&+R_{s, K}(1, \beta ; \lambda, 0 ; q)-R_{s, K}(1,1-\beta ; 1-\lambda, 0 ; q)-\zeta(s, \beta)
\end{aligned}
$$

and (8.15) instead of (8.11) and (8.14) respectively. The proof of Theorem 4 is complete.

9. Proof of Theorem 5 and its corollary. The scheme of the proof of Theorem 5 is almost the same as that of Theorem 0 . By setting $q_{h / k}=$ $e(h / k) q$ with $q=e^{-t}$ it is seen that

$$
\begin{aligned}
\log \left(e(\mu) q_{h / k}^{\alpha} ; q_{h / k}\right)_{\infty} & =-\sum_{l=0}^{\infty} \sum_{n=1}^{\infty} n^{-1} e(\mu n) q_{h / k}^{(\alpha+l) n} \\
= & -\sum_{l=0}^{\infty} \sum_{m=0}^{\infty} \sum_{j=1}^{k}(j+k m)^{-1} e((j+k m) \mu) \\
& \times e((\alpha+l)(j+k m) h / k) q^{(\alpha+l)(j+k m)} \\
= & -k^{-1} \sum_{j=1}^{k} e((\alpha h+\mu k) j / k) \\
& \times \sum_{l, m=0}^{\infty}(j / k+m)^{-1} e(l h j / k) e((\alpha h+\mu k) m) q^{(\alpha+l)(j+k m)}
\end{aligned}
$$

where we write $n=j+k m$ with $j=1, \ldots, k$ and $m=0,1, \ldots$ for the second equality. The last inner double sum is transformed by (6.2), and this gives

$$
\log \left(e(\mu) q_{h / k}^{\alpha} ; q_{h / k}\right)_{\infty}=-k^{-1} \sum_{j=1}^{k} e((\alpha h+\mu k) j / k) I_{j / k}(k t),
$$

where

$$
I_{\lambda}(z)=\frac{1}{2 \pi i} \int_{(c)} \Gamma(w) \phi(w, \alpha, \lambda h) \phi(1+w, \lambda, \alpha h+\mu k) z^{-w} d w
$$

for $|\arg z|<\pi / 2$, with a constant $c>1$.

Let $N$ be any positive integer, and $c_{N}$ the constant satisfying $-N<c_{N}<$ $-N+1$. Then in view of the vertical order estimate for $\phi(w, \alpha, \lambda)$ stated in Section 6, we can move the path of integration in (9.2) from $(c)$ to $\left(c_{N}\right)$, passing over the (possible) poles at $w=1$ and $w=-n(n=0,1, \ldots, N-1)$. 
By using the Laurent series expansions (near $w=0$ )

$$
\begin{gathered}
\Gamma(w)=w^{-1}\left\{1-\gamma_{0} w+O\left(w^{2}\right)\right\}, \quad \phi(w, \alpha, \lambda h)=-\mathcal{B}_{1}+\mathcal{D} w+O\left(w^{2}\right), \\
\phi(1+w, \lambda, \alpha h+\mu k)=w^{-1}\left\{\mathcal{B}_{0}+\mathcal{C} w+O\left(w^{2}\right)\right\}, \\
z^{-w}=1-(\log z) w+O\left(w^{2}\right),
\end{gathered}
$$

where we write $\mathcal{B}_{0}=\mathcal{B}_{0}(\lambda, e(\alpha h+\mu k)), \mathcal{B}_{1}=\mathcal{B}_{1}(\alpha, e(\lambda h)), \mathcal{C}=\mathcal{C}(\lambda, e(\alpha h+$ $\mu k)$ ) and $\mathcal{D}=\mathcal{D}(\alpha, e(\lambda h))$ for brevity, the residue of the (possible) double pole at $w=0$ is in particular computed as $\gamma_{0} \mathcal{B}_{0} \mathcal{B}_{1}+\mathcal{B}_{0} \mathcal{D}-\mathcal{B}_{1} \mathcal{C}+\mathcal{B}_{0} \mathcal{B}_{1} \log z$. We therefore obtain

$$
\begin{aligned}
I_{\lambda}(z)= & \mathcal{B}_{0}(\alpha, e(\lambda h)) \phi(2, \lambda, \alpha h+\mu k) z^{-1} \\
& +\mathcal{B}_{0} \mathcal{B}_{1} \log z+\mathcal{B}_{0}\left(\gamma_{0} \mathcal{B}_{1}+\mathcal{D}\right)-\mathcal{B}_{1} \mathcal{C} \\
& +\sum_{n=1}^{N-1} \frac{(-1)^{n} \mathcal{B}_{n}(\lambda, e(\alpha h+\mu k)) \mathcal{B}_{n+1}(\alpha, e(\lambda h))}{n(n+1) !} z^{n}+O\left(|z|^{N}\right)
\end{aligned}
$$

as $z \rightarrow 0$ in the sector $|\arg z| \leq \pi / 2-\delta$ with any small $\delta>0$, where the implied $O$-constant depends at most on $N, h, \alpha, \mu$ and $\delta$. Here the last error estimate follows similarly to the proof of (2.4). The assertion (5.5) is thus concluded from (9.1) and (9.3), where the coefficient of $(k t)^{-1}$ is evaluated by noting $(2.7)$.

For the proof of Corollary 5.1 we need

Lemma 9. Let $h$ and $k$ be relatively prime integers with $k \geq 1$. Then

$$
\sum_{j=1}^{k} \mathcal{B}_{n}(x, c e(h j / k))=k^{n} \mathcal{B}_{n}\left(x / k, c^{k}\right)
$$

for any integer $n \geq 0$ and any complex $c \neq 0$.

Proof. Equate the coefficients of the Taylor series (near $z=0$ ) for both sides of the identity

$$
\sum_{j=1}^{k} \frac{z e^{x z}}{c e(h j / k) e^{z}-1}=\frac{k z e^{x z}}{c^{k} e^{k z}-1} .
$$

Proof of Corollary 5.1. Evaluation of $\mathcal{A}_{-1}(1,0, h ; k)$ is immediate by noting that $\zeta(2)=\pi^{2} / 6$. Next by using (7.1) and (7.2) it is seen that

$$
\begin{aligned}
\mathcal{A}_{0}(1,0, h ; k)= & k^{-1} \sum_{j=1}^{k}\left[\gamma_{0}\left\{\mathcal{B}_{1}(0, e(h j / k))+1\right\}+e(h j / k) \mathcal{D}(1, e(h j / k))\right. \\
& \left.-\left\{\mathcal{B}_{1}(0, e(h j / k))+1\right\} \mathcal{C}(j / k, 1)\right],
\end{aligned}
$$

where the facts

$$
e(\lambda h) \mathcal{D}(1, e(\lambda h))=\zeta_{\lambda h}^{\prime}(0) \quad \text { and } \quad \mathcal{C}(\lambda, 1)=-\psi(\lambda)
$$


follow from the definitions (cf. [Er1, 1.10, (9)]). Then in view of Lemma 9, (2.8) and the relations

$$
\sum_{j=1}^{k} \zeta_{h j / k}(s)=k^{1-s} \zeta(s) \text { and } \sum_{j=1}^{k} \psi(j / k)=-\gamma_{0} k-k \log k,
$$

we obtain (5.3) upon noting that $\zeta(0)=-1 / 2$ and $\zeta^{\prime}(0)=-(1 / 2) \log 2 \pi$ (cf. [Er1, 1.12, (18)]). Evaluation of $\mathcal{A}(1,0, h ; k)$ is similar but less involved. The last assertion (5.4) is immediate by using (7.1) and (7.2) again. This completes the proof.

\section{References}

[An] G. E. Andrews, The Theory of Partitions, Addison-Wesley, New York, 1976.

[Ap1] T. M. Apostol, On the Lerch zeta function, Pacific J. Math. 1 (1951), 161-167.

[Ap2] - Modular Functions and Dirichlet Series in Number Theory, 2nd ed., Springer, New York, 1990.

[Be1] B. C. Berndt, Modular transformations and generalizations of several formulae of Ramanujan, Rocky Mountain J. Math. 7 (1977), 147-189.

[Be2] -, Ramanujan's Notebooks, Part II, Springer, New York, 1989.

[Be3] - Some asymptotic formulas for q-series found in Ramanujan's third notebook and "lost notebook", Indian J. Math. 32 (1990), 179-185.

[Be4] -, Ramanujan's Notebooks, Part III, Springer, New York, 1991.

[Be5] -, Ramanujan's Notebooks, Part IV, Springer, New York, 1994.

[EC] M. Eie and K.-W. Chen, A theorem on zeta functions associated with polynomials, Trans. Amer. Math. Soc. 351 (1999), 3217-3228.

[Er1] A. Erdélyi (ed.), W. Magnus, F. Oberhettinger and F. G. Tricomi, Higher Transcendental Functions, Vol. I, McGraw-Hill, New York, 1953.

[Er2] - Tables of Integral Transforms, Vol. I, McGraw-Hill, New York, 1954.

[GR] G. Gasper and M. Rahman, Basic Hypergeometric Series, Encyclopedia Math. Appl. 35, Cambridge Univ. Press, Cambridge, 1990.

[GM1] B. Gordon and R. J. McIntosh, Some eighth order mock theta functions, J. London Math. Soc. (2) 62 (2000), 321-335.

[GM2] -, -, Modular transformations of Ramanujan's fifth and seventh order mock theta functions, in preparation.

[Ka1] M. Katsurada, Power series with the Riemann zeta-function in the coefficients, Proc. Japan Acad. Ser. A 72 (1996), 61-63.

[Ka2] -, On Mellin-Barnes type of integrals and sums associated with the Riemann zeta-function, Publ. Inst. Math. (Beograd) (N.S.) 62(76) (1997), 13-25.

[Ka3] - An application of Mellin-Barnes' type integrals to the mean square of Lerch zeta-functions, Collect. Math. 48 (1997), 137-153.

[Ka4] - An application of Mellin-Barnes type of integrals to the mean square of $L$ functions, Liet. Mat. Rink. 38 (1998), 98-112.

[Ka5] - Power series and asymptotic series associated with the Lerch zeta-function, Proc. Japan Acad. Ser. A 74 (1998), 167-170.

[Ka6] - Rapidly convergent series representations for $\zeta(2 n+1)$ and their $\chi$-analogue, Acta Arith. 90 (1999), 79-89. 
[Ka7] M. Katsurada, On an asymptotic formula of Ramanujan for a certain theta-type series, ibid. 97 (2001), 157-172.

[Mc1] R. McIntosh, Some asymptotic formulae for q-hypergeometric series, J. London Math. Soc. (2) 51 (1995), 120-136.

[Mc2] -, Asymptotic transformations of q-series, Canad. J. Math. 50 (1998), 412-425.

[Mc3] - Some asymptotic formulae for q-shifted factorials, Ramanujan J. 3 (1999), 205-214.

[Me1] G. Meinardus, Asymptotische Aussagen über Partitionen, Math. Z. 59 (1954), 388-398.

[Me2] -

[Mo] D. S. Moak, The q-analogue of Stirling's formula, Rocky Mountain J. Math. 14 (1984), 403-413.

[Ra1] S. Ramanujan, Notebooks, 2 volumes, Tata Institute of Fundamental Research, Bombay, 1957.

[Ra2] - The Lost Notebook and Other Unpublished Papers, Narosa, New Delhi, 1988.

[UN] K. Ueno and M. Nishizawa, Quantum groups and zeta-functions, in: Quantum Groups: Formalism and Applications, Proc. XXXth Karpacz Winter School, J. Lukierski, Z. Popowicz and J. Sobczyk (eds.), Polish Scientific Publishers, 1994, 115-126.

[Wa] G. N. Watson, The final problem: an account of the mock theta functions, J. London Math. Soc. 11 (1936), 55-80.

Mathematics, Hiyoshi Campus

Keio University

Hiyoshi 4-1-1, Kuohoku-ku

Yokohama 223-8521, Japan

E-mail: masanori@math.hc.keio.ac.jp

Received on 27.11.2001

and in revised form on 7.6.2002 